Louisiana State University

LSU Digital Commons

Faculty Publications

Department of Physics \& Astronomy

$1-27-2021$

\title{
Helical magnetic order and Fermi surface nesting in noncentrosymmetric ScFeGe
}

\author{
Sunil K. Karna \\ Louisiana State University \\ D. Tristant \\ Cain Department of Chemical Engineering \\ J. K. Hebert \\ Louisiana State University \\ G. Cao \\ Louisiana State University \\ R. Chapai \\ Louisiana State University
}

See next page for additional authors

Follow this and additional works at: https://digitalcommons.Isu.edu/physics_astronomy_pubs

\section{Recommended Citation}

Karna, S., Tristant, D., Hebert, J., Cao, G., Chapai, R., Phelan, W., Zhang, Q., Wu, Y., Dhital, C., Li, Y., Cao, H., Tian, W., Dela Cruz, C., Aczel, A., Zaharko, O., Khasanov, A., McGuire, M., Roy, A., Xie, W., Browne, D., Vekhter, I., Meunier, V., Shelton, W., Adams, P., Sprunger, P., Young, D., Jin, R., \& Ditusa, J. (2021). Helical magnetic order and Fermi surface nesting in noncentrosymmetric ScFeGe. Physical Review B, 103 (1) https://doi.org/10.1103/PhysRevB.103.014443

This Article is brought to you for free and open access by the Department of Physics \& Astronomy at LSU Digital Commons. It has been accepted for inclusion in Faculty Publications by an authorized administrator of LSU Digital Commons. For more information, please contact ir@lsu.edu. 


\section{Authors}

Sunil K. Karna, D. Tristant, J. K. Hebert, G. Cao, R. Chapai, W. A. Phelan, Q. Zhang, Y. Wu, C. Dhital, Y. Li, H. B. Cao, W. Tian, C. R. Dela Cruz, A. A. Aczel, O. Zaharko, A. Khasanov, M. A. McGuire, A. Roy, W. Xie, D. A. Browne, I. Vekhter, V. Meunier, W. A. Shelton, P. W. Adams, P. T. Sprunger, D. P. Young, R. Jin, and J. F. Ditusa 


\title{
Helical magnetic order and Fermi surface nesting in noncentrosymmetric ScFeGe
}

\author{
Sunil K. Karna $\odot,{ }^{1,2,{ }^{*}}$ D. Tristant ${ }^{3}$ J. K. Hebert,,${ }^{1, \dagger}$ G. Cao,${ }^{1,4}$ R. Chapai, ${ }^{1}$ W. A. Phelan,,${ }^{1, \dagger}$ Q. Zhang,,${ }^{1,5}$ Y. Wu,,${ }^{1,5}$ C. Dhital, ${ }^{1,6}$ \\ Y. Li,${ }^{1}$ H. B. Cao, ${ }^{5}$ W. Tian, ${ }^{5}$ C. R. Dela Cruz,${ }^{5}$ A. A. Aczel,${ }^{5}$ O. Zaharko, ${ }^{7}$ A. Khasanov, ${ }^{8}$ M. A. McGuire, ${ }^{9}$ A. Roy, ${ }^{10}$

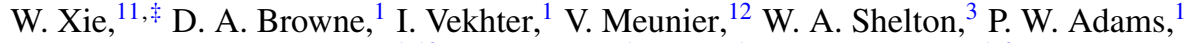 \\ P. T. Sprunger, ${ }^{1,10}$ D. P. Young, ${ }^{1}$ R. Jin, ${ }^{1}$ and J. F. DiTusa ${ }^{1, \S}$ \\ ${ }^{1}$ Department of Physics \& Astronomy, Louisiana State University, Baton Rouge, Louisiana 70803, USA \\ ${ }^{2}$ Department of Physics \& Center for Materials Research, Norfolk State University, Norfolk, Virginia 23504, USA \\ ${ }^{3}$ Cain Department of Chemical Engineering, Louisiana State University, Baton Rouge, Louisiana 70803, USA \\ ${ }^{4}$ Materials Genome Institute and Department of Physics, Shanghai University, Shanghai 200444, China \\ ${ }^{5}$ Neutron Scattering Division, Oak Ridge National Laboratory, Oak Ridge, Tennessee 37831, USA \\ ${ }^{6}$ Department of Physics, Kennesaw State University, Marietta, Georgia 30060, USA \\ ${ }^{7}$ Laboratory for Neutron Scattering and Imaging, PSI, CH-5232 Villigen, Switzerland \\ ${ }^{8}$ Mössbauer Effect Data Center, University of North Carolina, Asheville, North Carolina 28804, USA \\ ${ }^{9}$ Materials Science and Technology Division, Oak Ridge National Laboratory, Oak Ridge, Tennessee 37831, USA \\ ${ }^{10}$ Center for Advanced Microstructures and Devices (CAMD), Louisiana State University, Baton Rouge, Louisiana 70803, USA \\ ${ }^{11}$ Department of Chemistry, Louisiana State University, Baton Rouge, Louisiana 70803, USA \\ ${ }^{12}$ Department of Physics, Applied Physics, and Astronomy, Rensselaer Polytechnic Institute, Troy, New York 12180, USA
}

(Received 18 September 2020; revised 9 December 2020; accepted 11 January 2021; published 27 January 2021)

\begin{abstract}
An investigation of the structural, magnetic, thermodynamic, and charge transport properties of noncentrosymmetric hexagonal $\mathrm{ScFeGe}$ reveals it to be an anisotropic metal with a transition to a weak itinerant incommensurate helimagnetic state below $T_{N}=36 \mathrm{~K}$. Neutron diffraction measurements discovered a temperature and field independent helical wave vector $\boldsymbol{k}=\left(\begin{array}{lll}0 & 0 & 0.193\end{array}\right)$ with magnetic moments of $0.53 \mu_{B}$ per $\mathrm{Fe}$ confined to the $a b$ plane. Density functional theory calculations are consistent with these measurements and find several bands that cross the Fermi level along the $c$ axis with a nearly degenerate set of flat bands just above the Fermi energy. The anisotropy found in the electrical transport is reflected in the calculated Fermi surface, which consists of several warped flat sheets along the $c$ axis with two regions of significant nesting, one of which has a wave vector that closely matches that found in the neutron diffraction. The electronic structure calculations, along with a strong anomaly in the $c$-axis conductivity at $T_{N}$, signal a Fermi surface driven magnetic transition, similar to that found in spin density wave materials. Magnetic fields applied in the $a b$ plane result in a metamagnetic transition with a threshold field of $\approx 6.7 \mathrm{~T}$ along with a sharp, strongly temperature dependent discontinuity and a change in sign of the magnetoresistance for in-plane currents. Thus, $\mathrm{ScFeGe}$ is an ideal system to investigate the effect of in-plane magnetic fields on a helimagnet with a $c$-axis propagation vector, where the relative strength of the magnetic interactions and anisotropies determine the topology and magnetic structure.
\end{abstract}

DOI: 10.1103/PhysRevB.103.014443

\section{INTRODUCTION}

The magnetism found in noncentrosymmetric (NCS) magnetic materials is compelling because of the topologically nontrivial nanoscopic magnetic structures discovered that offer potential for new magnetic information manipulation and storage technologies [1-10]. For example, $\mathrm{MnSi}$, which

\footnotetext{
*skkarna@nsu.edu

${ }^{\dagger}$ Present address: Los Alamos National Laboratory, Los Alamos, Mail Stop E574, Los Alamos, New Mexico 87545, USA.

ॠPresent address: Department of Chemistry and Chemical Biology, Rutgers University, Piscataway, New Jersey 08854, USA.

${ }^{\S}$ Present address: Department of Physics, Indiana UniversityPurdue University Indianapolis, Indianapolis, Indiana 46202, USA; jfditusa@iu.edu
}

has a NCS crystal structure that also lacks mirror symmetry so that it is chiral, was discovered to host a skyrmion lattice (Bloch type), an ordered array of topologically stable knots of spin structure [2-4,11,12]. In contrast, the NCS and achiral $\mathrm{GaV}_{4} \mathrm{~S}_{8}$ and $\mathrm{VOSe}_{2} \mathrm{O}_{5}$ display Néel-type skyrmion lattice states that may be controllable via electric fields $[13,14]$. The cause of these interesting behaviors is the Dzyaloshinskii-Moriya (DM) interaction, which occurs only in NCS materials resulting in topologically nontrivial magnetic structures, such as the magnetic $B 20$ materials, including $\mathrm{FeGe}, \mathrm{Fe}_{1-x} \mathrm{Co}_{x} \mathrm{Si}$, and $\mathrm{MnGe}[4,15,16]$. While the cubic symmetry appears to promote skyrmion lattice formation, hexagonal chiral magnetic materials, such as $\mathrm{Cr}_{1 / 3} \mathrm{NbS}_{2}$ and $\mathrm{Mn}_{1 / 3} \mathrm{NbS}_{2}$, display helimagnetism with a transition to unusual magnetic phases, including the chiral magnetic soliton lattice [6-8].

Although the DM interaction is expected to be significant in all NCS materials, thus far there have been very few 
discoveries of topologically interesting magnetic phases in bulk achiral materials outside of those caused by demagnetization fields. The DM interaction stems from spinorbit coupling and is antisymmetric, favoring perpendicular arrangements of magnetic moments [17]. The role that the DM interaction plays in polar NCS compounds, ones that retain mirror symmetry, is not as well explored. The lack of a left/right asymmetry in the crystal structure of polar NCS systems would tend to rule out topological effects, despite the presence of the DM interaction. Topological features may, however, be present in polar NCS helimagnets, since the magnetic ordering itself breaks mirror symmetry. The discovery of such features in additional NCS magnets would also be important because of the interest in magnetic topological structures, such as skyrmions, for possible technological applications (skyrmtronics) $[18,19]$.

Helimagnetic ordering in hexagonal structured materials has been of high recent interest. The application of a magnetic field perpendicular to the propagation vector causes a distortion of the helical order leading to interesting magnetic structures, as moments tend to align with the external field. The sequence of magnetic structures that results as the system evolves to the field polarized state depends heavily on the symmetry of the crystal lattice, in particular whether the space group is chiral or achiral, and the relative strength of important interactions. These include the exchange interaction, the DM interaction, the magnetic anisotropy, and the dipole-dipole interactions through the local demagnetization fields. The most interesting systems are those where the magnetic anisotropy is sufficient to confine the moments to the $a b$ plane, even when exposed to in-plane fields. For systems that are dominated by the DM interaction, such that one chirality or handedness of the helical state is highly preferred, the magnetic structure evolves to one where regions of magnetic moments aligned with $H$ are separated by $2 \pi$ chiral domain walls. This state is known as the magnetic soliton lattice [6,8]. Further increases in $H$ eventually cause a breakdown of the topological protection afforded this state, so that it transforms to the topologically trivial field-polarized state.

In cases where the DM interaction is not sufficient to cause a significant energy difference between the two chiralities, such as in achiral NCS magnets, a similar distortion of the helimagnetic structure results with the application of small in-plane fields. However, a first order metamagnetic transition at $H_{\mathrm{MM}} \sim 1 / 2 H_{\text {sat }}$, where $H_{\text {sat }}$ is the saturation field, leads to a magnetic fan state. This transition is usually hysteretic, as a large fraction of magnetic moments rearrange at $H_{\mathrm{MM}}$. The fan state consists of alternating regions along the $c$ axis of left- and right-handed twists forming a lattice of kinks, where the helicity reverses and is thus a topologically trivial state. Further increases in field result in a smooth reduction in turn angles between kinks until the system reaches full polarization. Recent investigations have discovered more complex magnetic structures including helifan states and spin-slip phases [20].

Here, we explore the physical properties of $\mathrm{ScFeGe}$, which crystallizes in the $\mathrm{Fe}_{2} \mathrm{P}$-type [ $\mathrm{ZrNiAl}$ structure type, symmetry group $P \overline{6} 2 m$ ] hexagonal crystal structure that is common among transition metal ternary compounds [21]. This crystal structure, shown in Fig. 1, is NCS but achiral, and is

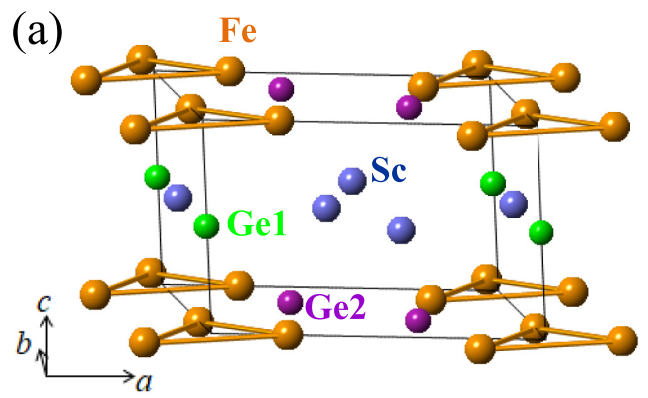

(b)

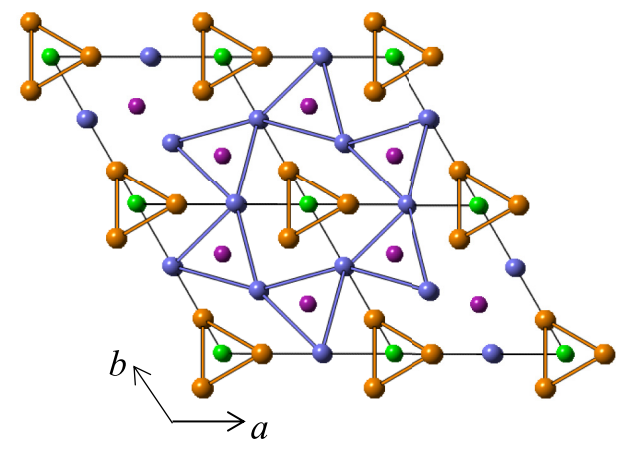

FIG. 1. Crystal structure. (a) Crystal structure of ScFeGe, highlighting the triangular network of $\mathrm{Fe}$ located at the $3 f$ site. (b) Two-dimensional view of the crystal structure along the $c$ axis displaying the distorted kagome lattice of Sc.

adopted by a number of magnetic materials, including several that display interesting magnetocaloric properties, such as $\mathrm{Fe}_{2} \mathrm{P}, \mathrm{MnFeP}_{1-x} \mathrm{Ge}_{x}$ [22], and $\mathrm{MnFeP}_{1-x} \mathrm{As}_{x}$ [23]. Those most heavily investigated contain two magnetic species, one on the $3 g$ site that is described as forming a distorted kagome lattice (the Sc site in Fig. 1), and one on the $3 f$ site (Fe) that forms well-separated triangular stacks. These materials tend to order ferromagnetically, where the magnetic phase transition is accompanied by a discontinuous and hysteretic change in the $c / a$ lattice constant ratio, making them good candidates for magnetocaloric applications. In contrast, $\mathrm{ScFeGe}$ has only a single species that is typically magnetic, $\mathrm{Fe}$, and is therefore a somewhat simpler system to explore the role of crystal symmetry on its magnetic structure and the possibility of topologically interesting spin textures. Very little is known about this compound outside of its crystal structure [24]. We discover that $\mathrm{ScFeGe}$ is a weak itinerant magnet, ordering into a helimagnetic state with an incommensurate wave vector of $\boldsymbol{k}=\left(\begin{array}{lll}0 & 0 & 0.193\end{array}\right)$ (in units of rlu) at $36 \mathrm{~K}$, caused by a Fermi surface instability. Interestingly, the cause of the helical ordering does not conform with the two most common origins for helimagnetism. Helical magnetism is associated most closely with either a significant DM interaction or with competing exchange interactions of comparable magnitudes and opposite signs. Instead, for $\mathrm{ScFeGe}$ electronic structure calculations reveal a significant nesting condition that matches the helical wave vector found in neutron scattering indicating a novel Fermi surface driven magnetic transition to a helically ordered state. Further, despite its itinerant character, we find that $\mathrm{ScFeGe}$ has many features in common with rare earth elemental metals, such as Dy, Ho, and $\mathrm{Tb}$. 


\section{EXPERIMENTAL DETAILS}

\section{A. Sample preparation}

Polycrystalline samples of $\mathrm{ScFeGe}$ were prepared via arcmelting techniques. High purity $(99.999 \%)$ starting materials consisting of the elements Sc, Fe, and Ge were melted on a water-cooled copper hearth in a $\mathrm{Zr}$-gettered ultrapure argon atmosphere. Each button was flipped and melted several times to ensure homogeneity. Only those polycrystalline samples of $\mathrm{ScFeGe}$ corresponding to a $1 \%$ or less calculated mass loss were employed for the single crystal growth. Five single-phase buttons that were produced in this manner were placed in a second copper hearth in the same Zr-gettered ultrapure argon atmosphere and arc melted for a second time to produce a rod for crystal growth employing the floatingzone method. Feed and seed rods were obtained from this arc-melted rod. A large single crystal of $\mathrm{ScFeGe}$ was synthesized in a floating-zone furnace-a Cannon FZ- MDH20020 2-mirror image furnace with $2 \times 1000 \mathrm{~W}$ lamps under Ar gas flow. A growth rate of $2 \mathrm{~mm} / \mathrm{h}$ was used to promote low mosaicity, and the seed and feed rods were counter-rotated at 12 to $14 \mathrm{rpm}$. The obtained cylindrical crystal with a diameter of about $2.5 \mathrm{~mm}$ was cut to a suitable size for magnetic, transport, heat capacity, and neutron diffraction measurements.

Powder x-ray diffraction (PXRD) measurements of a crushed single crystal and our polycrystalline samples were carried out on a PANalytical Empyrean multistage x-ray diffractometer with $\mathrm{Cu} \mathrm{K} \alpha_{1}$ radiation $(\lambda=1.54059 \AA)$. The resulting $\mathrm{x}$-ray diffraction patterns were analyzed using the General Structure Analysis System (GSAS) program [25] following the Rietveld profile refining method [26]. The refinement confirmed a hexagonal crystal structure (Fig. 1) with space group $P \overline{6} 2 m$. The lattice parameters for the crushed single crystal were $a=6.5410$ (4) $\AA$ and $c=3.8905$ (3) Å (Table $\mathrm{S} 1$ in the Supplemental Material [27]). For the polycrystalline samples, the lattice constants varied somewhat, being as large as $a=6.5451$ (4) $\AA$ and $c=3.8938$ (2) $\AA$, with the variation consistent with increases in the Ge content of the samples (see below). These measurements were followed by single crystal x-ray diffraction characterization performed on single crystals mounted onto a glass fiber tip using epoxy, attached to a goniometer head via the ends of brass pins and placed on a Nonius Kappa CCD x-ray diffractometer equipped with Mo$\mathrm{K} \alpha$ radiation $(\lambda=0.71073 \AA)$. The single crystal diffraction confirmed the crystal structure determined from PXRD and provided orientation of the crystals for further characterization.

Chemical analysis was performed using an electron probe x-ray microanalyzer (EPMA) with a JEOL JSX-8230 analyzer located at the Shared Instrumentation Facility (SIF) at Louisiana State University (LSU). This instrument allows simultaneous measurement via wavelength dispersive spectroscopy (WDS) and energy dispersive spectroscopy (EDS) techniques. EDS and WDS provide a chemical composition of the single crystals as $\mathrm{Sc}_{1.00} \mathrm{Fe}_{1.02(2)} \mathrm{Ge}_{0.99(2)}$, hereafter referred to as $\mathrm{ScFeGe}$. In addition, the polycrystalline samples were found to typically contain as much as $8 \%$ excess Ge, creating a negative chemical pressure as indicated by slightly increased lattice constants. The excess Ge content and unit cell volume correlated well with an increased magnetic ordering temperature (see below).

\section{B. Magnetic, charge transport, and thermodynamic measurements}

Magnetization and magnetic susceptibility measurements were carried out on both polycrystalline and single crystal samples in a Quantum Design (QD) magnetic property measurement system (MPMS) superconducting quantum interference device (SQUID) magnetometer with a 7-T superconducting magnet. For the single crystals, a magnetic field was applied either parallel or perpendicular to the crystallographic $c$ axis. The electrical resistivity and magnetoresistance were measured on polished single crystals with contacts formed via conductive epoxy (Epotek H20E) and thin platinum wire. These were standard four-terminal $d c$ resistance measurements with a current of $4 \mathrm{~mA}$ applied parallel to the $a$ and $c$ axes of the crystals. Data were collected in a QD physical property measurement system (PPMS) with a $14 \mathrm{~T}$ superconducting magnet. The specific heat capacity was measured using a time-relaxation method in a QD PPMS between 2 and $100 \mathrm{~K}$.

\section{Neutron diffraction}

Neutron powder diffraction (NPD) measurements were performed using the HB-2A neutron powder diffractometer at the high flux isotope reactor (HFIR) at Oak Ridge National Laboratory (ORNL). A five-gram polycrystalline sample of $\mathrm{ScFeGe}$ was sealed in an aluminum sample can in a helium atmosphere. The temperature dependence of the diffraction profiles in zero and in an applied magnetic field of $4 \mathrm{~T}$ was measured in the temperature range between 2 $300 \mathrm{~K}$ using a vertical-field cryomagnet. An incident neutron beam with a wavelength of $2.41 \AA$, defined by a Ge (113) crystal monochromator, was employed for these measurements. In addition, the crystal and magnetic structure of a single crystal taken from the floating zone sample were investigated by neutron diffraction measurements using the four-circle diffractometer HB-3A. Single crystal diffraction measurements under applied magnetic field were also performed using the HB-1A triple-axis spectrometer at ORNL using a vertical cryomagnet and employing a wavelength of $2.367 \AA$ A. Data collected at HB-3A included 105 reflections taken at $T=4$ and $285 \mathrm{~K}$ using a wavelength of $1.546 \AA$ (Si-220 monochromator) [28]. Both the powder and single crystal neutron experiments reproduced the crystal structure determined from our $\mathrm{x}$-ray diffraction. The neutron powder diffraction patterns were analyzed using the GSAS program, whereas single-crystal nuclear and magnetic structural refinements were performed with the FULLPROF suite [29].

Figure 2(a) displays the neutron powder diffraction patterns (crosses) that were taken at $285 \mathrm{~K}$ along with the results of the refinement (solid lines), with the difference plotted at the bottom. The refinement was conducted assuming a hexagonal symmetry with a space group of $P \overline{6} 2 m$ and taking the pseudo-Voigt function for the peak profiles. The refinement indicated that several low intensity peaks originated from a small impurity phase identified as $\mathrm{Sc}_{4} \mathrm{Fe}_{4} \mathrm{Ge}_{6.6}$ as well as 


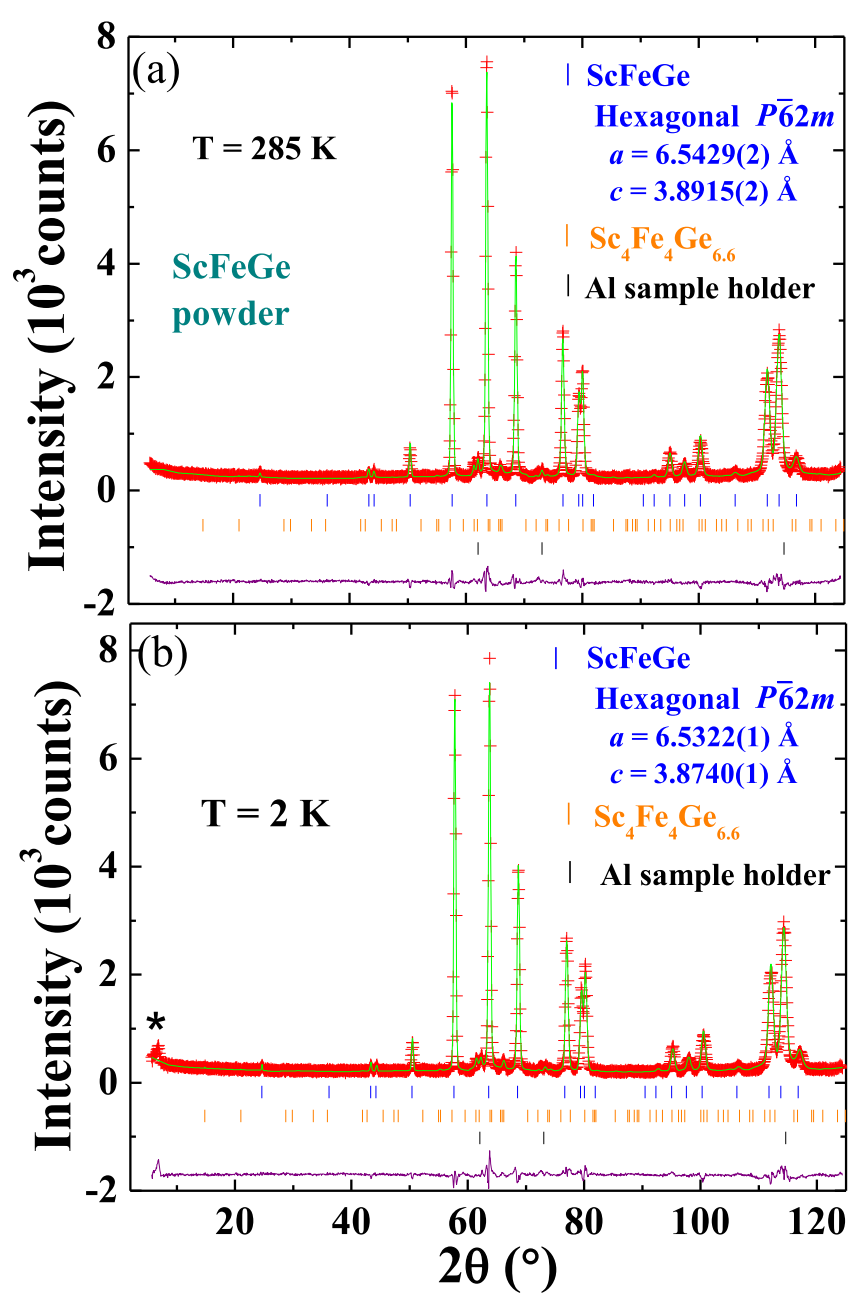

FIG. 2. Neutron powder diffraction. High-resolution neutron powder-diffraction data (red crosses) taken at (a) $285 \mathrm{~K}$ and (b) $2 \mathrm{~K}$. The green line is the result of a structural refinement which indicated an $\mathrm{Fe}_{2} \mathrm{P}$ hexagonal crystal structure having the $P \overline{6} 2 \mathrm{~m}$ space group with peak positions indicated by the blue vertical lines. Several low intensity peaks were identified as originating from a small $\mathrm{Sc}_{4} \mathrm{Fe}_{4} \mathrm{Ge}_{6.6}$ impurity phase and from the Al sample holder. The refinement includes these contributions with peak positions indicated by the orange and black vertical lines. The remaining difference between the data and the fit is included as a purple line at the bottom of the figure. The asterisk marked in (b) is a magnetic peak. Data were collected at the HB-2A beam line at ORNL.

the Al sample holder. The low intensity impurity phase of $\mathrm{Sc}_{4} \mathrm{Fe}_{4} \mathrm{Ge}_{6.6}$ and $\mathrm{Al}$ sample holder have also been taken into consideration in the refinement. The solid vertical lines mark the calculated positions of the Bragg reflections of the proposed crystalline structure, the $\mathrm{Sc}_{4} \mathrm{Fe}_{4} \mathrm{Ge}_{6.6}$ impurity phase, and the Al sample holder. Table S2 in the Supplemental Material [27] presents the refinement results of $\mathrm{ScFeGe}$ obtained at $285 \mathrm{~K}$. No structural change with cooling is detected, as demonstrated by the diffraction pattern obtained at $2 \mathrm{~K}$ [Fig. 2(b)]. Neutron diffraction experiments were also performed at the single-crystal neutron diffractometer ZEBRA at the Swiss Neutron Spallation Source, Paul Scherrer Institut (PSI) in Switzerland. A Ge (311) monochromator was chosen to obtain neutrons with the wavelength of $1.178 \AA$, and a helium cryostat was used to achieve a base temperature of $2 \mathrm{~K}$.

\section{Mössbauer measurements}

Mössbauer spectra were collected on a polycrystalline sample of ScFeGe with a Wissel spectrometer in the constantacceleration mode over a range of temperatures between 8 and $295 \mathrm{~K}$. A cobalt source in a rhodium matrix was used for the measurements, and $\alpha$-Fe was employed as a reference material for the isomer shift. The spectra were fit using a static Hamiltonian with a Lorentzian line shape. The fitting procedure adjusts parameters of the hyperfine Hamiltonian listed in the Supplemental Material [27], consisting of the isomer shift (IS), the quadrupole splitting (QS), the hyperfine field (HI), the electric field gradient (EFG) tensor asymmetry parameter $(\eta)$, the angle between the hyperfine field and main axis of the EFG $(\Theta)$, as well as the background count level, intensity of the absorption spectrum and the experimental linewidth (W). This approach allows the calculation of the expected spectral shape for any combination of magnetic dipole and electric quadrupole components contributing to the hyperfine Hamiltonian. It allows a seamless description of a pure quadrupole spectrum above the magnetic ordering temperature and a combined magnetic and quadrupole splitting below the critical point. This experiment was performed using the "thin absorber" technique, where the sample area density is less that $10 \mathrm{mg}$ of $\mathrm{Fe} / \mathrm{cm}^{2}$ to guarantee a Lorentzian line shape of the spectral components. The best fit of the calculated spectrum to the experimental data was determined via the $\chi^{2}$ criterion.

\section{E. X-ray absorption spectroscopy measurements}

Scandium, iron, and germanium $K$-edge x-ray absorption near edge structure (XANES) spectroscopic measurements were performed at the J. Bennett Johnston, Sr., Center for Advanced Microstructures and Devices (CAMD) electron storage ring at Louisiana State University. Measurement details are very similar to those provided in the previous reports [30,31]. All measurements were made in transmission mode, and the data analysis was performed with Athena [32].

\section{F. First-principles calculations}

We performed first-principles calculations based on density functional theory (DFT). Calculations of the structural, electronic, and magnetic properties of bulk ScFeGe were performed using the Vienna $a b$ initio simulation package (VASP) [33-36]. Ion cores were modeled with projector augmented wave (PAW) pseudopotentials [37]. The valence $3 s, 3 p, 3 d$, and $4 s$ states of scandium, the $3 p, 3 d$, and $4 s$ states of iron, and the $3 d, 4 s$, and $4 p$ states of germanium are treated explicitly. A plane-wave basis energy cutoff of $520 \mathrm{eV}$ and a Gaussian smearing of $0.01 \mathrm{eV}$ were found to yield converged total energy and forces. For comparison purposes, we used the Perdew, Burke, and Ernzerhof (PBE) functional [38], and we included van der Waals (vdW) interactions in our calculations. We also used the vdW density functional (vdW-DF), vdW-DF2, optPBE-vdW, optB88-vdW, and optB86b-vdW schemes, as well as DFT-D2 and DFT-D3 [39-42]. The spin-orbit coupling (SOC) was included in the 

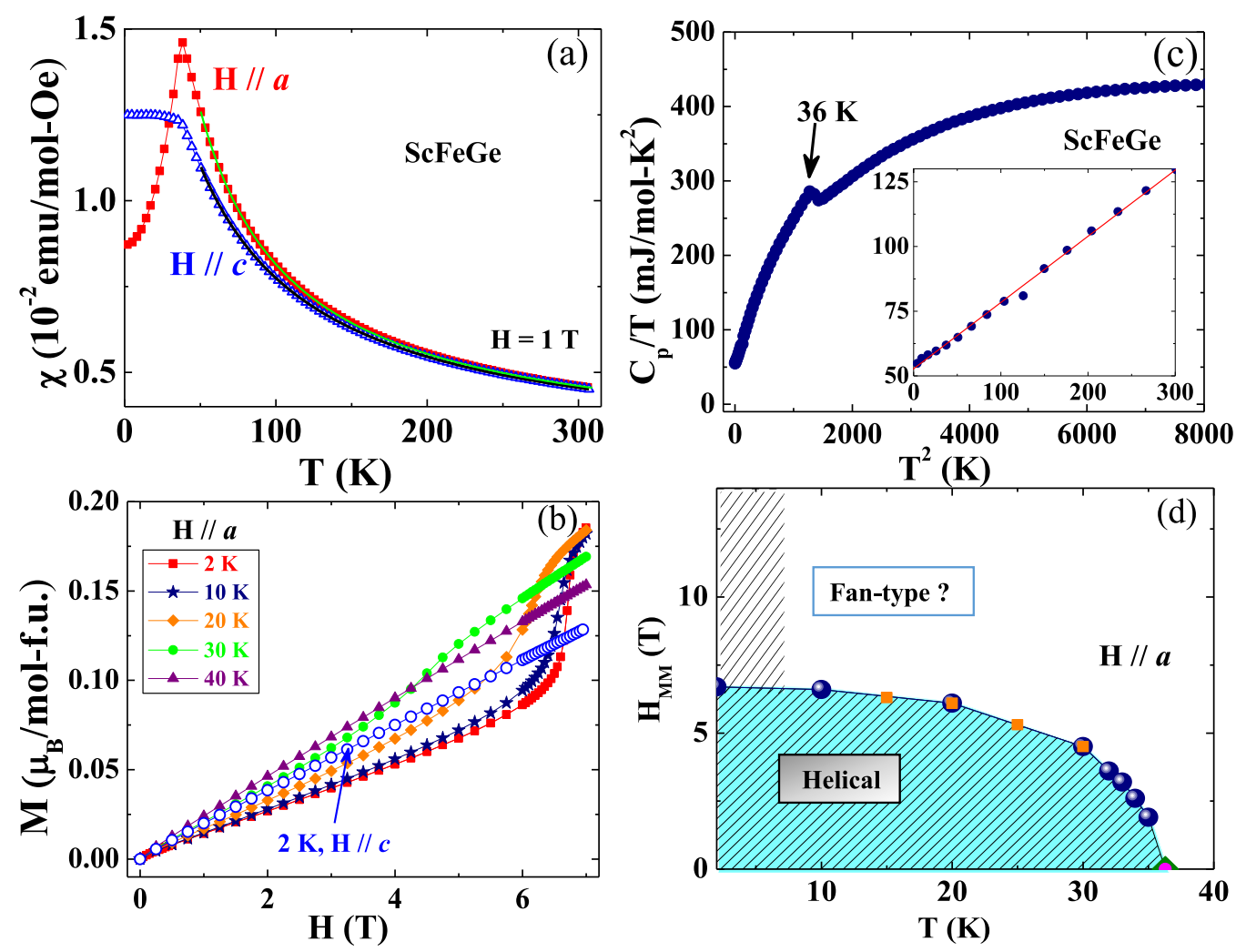

FIG. 3. Magnetic susceptibility, magnetization, and specific heat capacity. (a) Temperature $T$, dependence of the magnetic susceptibility $\chi$, with magnetic field $H$, oriented parallel to the $a$ and $c$ crystallographic axes. (b) $H$ dependence of the magnetization $M$ for the same field orientations. (c) Specific heat capacity divided by $T, C_{p}(T) / T$, vs $T^{2}$ at zero field. Inset: Low temperature region shown on an expanded scale. The solid line is the best fit of a linear form to the data. (d) Proposed magnetic phase diagram of ScFeGe as a function of temperature $T$ and magnetic field $H$ applied parallel to the crystallographic $a$ axis. The metamagnetic transition observed in $M(H)$ [Fig. 3(b)] is plotted in the phase diagram as solid blue spheres, and the magnetic ordering temperature is represented by a green triangle and magenta circle obtained, respectively, from the neutron diffraction and specific heat measurements. The orange rectangles indicate the transition from negative to positive MR for $T>10 \mathrm{~K}$ observed in $\Delta \rho_{a} / \rho_{a}(0)$ [Figs. 4(b) and 4(d)] whereas the hatched region in the phase diagram indicates a positive magnetoresistance.

self-consistent calculations. All atoms, as well as the cell, were relaxed to force a cutoff of $10^{-2} \mathrm{eV} \AA^{-1}$ with an electronic energy convergence threshold of $10^{-6} \mathrm{eV}$. The $k$-point sampling was based on a $\Gamma$-centered grid for all calculations. To optimize the primitive cells we used a $(10 \times 10 \times 10)$ grid and a $(7 \times 7 \times 7)$ grid for the supercells. Maximally-localized Wannier functions based on Sc- $d$, Fe- $d$, and Ge- $p$ orbitals were generated using the Wannier90 code $[43,44]$. Wanniertools code is used to calculate the surface state spectrum [45].

\section{EXPERIMENTAL RESULTS}

We begin the discussion of the magnetic properties of $\mathrm{ScFeGe}$ by presenting the temperature dependence of the magnetic susceptibility $\chi$ of a single crystal for $H=1 \mathrm{~T}$, parallel and perpendicular to the $c$ axis in Fig. 3(a). The sharp peak in $\chi$ at $T_{N}=36 \mathrm{~K}$, when $H$ is parallel to the $a$ axis, and the saturation of $\chi$ below this same temperature for $H \| c$ axis, indicate a magnetic transition at this temperature. A fit to the modified Curie-Weiss (CW) law, $\chi=$ $\chi_{0}+C /\left(T-\Theta_{W}\right)$, where $C$ is the Curie constant, $\Theta_{W}$ is the Weiss temperature, and $\chi_{0}$ the $T$-independent contributions to $\chi$, above $50 \mathrm{~K}$ yields $\Theta_{W}=-29.8(2) \mathrm{K}$ for $H \| c$ axis and $\Theta_{W}=-10.49(7) \mathrm{K}$ for $H \| a$ axis. These indicate an antiferromagnetic interaction of the magnetic moments. The best fit resulted in $C=0.666(2) \mathrm{emu} \mathrm{K} / \mathrm{mol}$ with an effective magnetic moment $\mu_{\text {eff }}=2.32(13) \mu_{B}$ for $H \| c$ axis and $C=$ $0.606(1) \mathrm{emu} \mathrm{K} / \mathrm{mol}, \mu_{\text {eff }}=2.21(8) \mu_{B}$ for $H \| a$ axis. These data indicate a moderate anisotropy for all temperatures, with a crossover below $28 \mathrm{~K}$. The transition temperature was found to vary between 36 and $38 \mathrm{~K}$ for our single crystal samples, while polycrystalline samples displayed a wider variation of $T_{N}$ up to $47 \mathrm{~K}$ with $T_{N}$ correlating well with the excess $\mathrm{Ge}$ content.

The magnetic field dependence of the magnetization $M$ of a single crystal sample is presented in Fig. 3(b) for fields up to $7 \mathrm{~T}$. As in $\chi(T), M(H)$ displays a moderate anisotropy. Interestingly, a sharp metamagnetic (MM) transition is observed at $H_{\mathrm{MM}}=6.7 \mathrm{~T}$ for $T=2 \mathrm{~K}$, when the field is oriented parallel to the $a$ axis but is absent when $H \|$ to the $c$ axis. $M(H)$ increases with field so that by $7 \mathrm{~T}, M=0.18 \mu_{B}$, far below the value of the fluctuating moment determined from the Curie-Weiss fit, which indicates that a much larger magnetic field is required to saturate the magnetization. The MM transition broadens and shifts to lower fields with increasing temperature, as highlighted in the proposed phase diagram of 

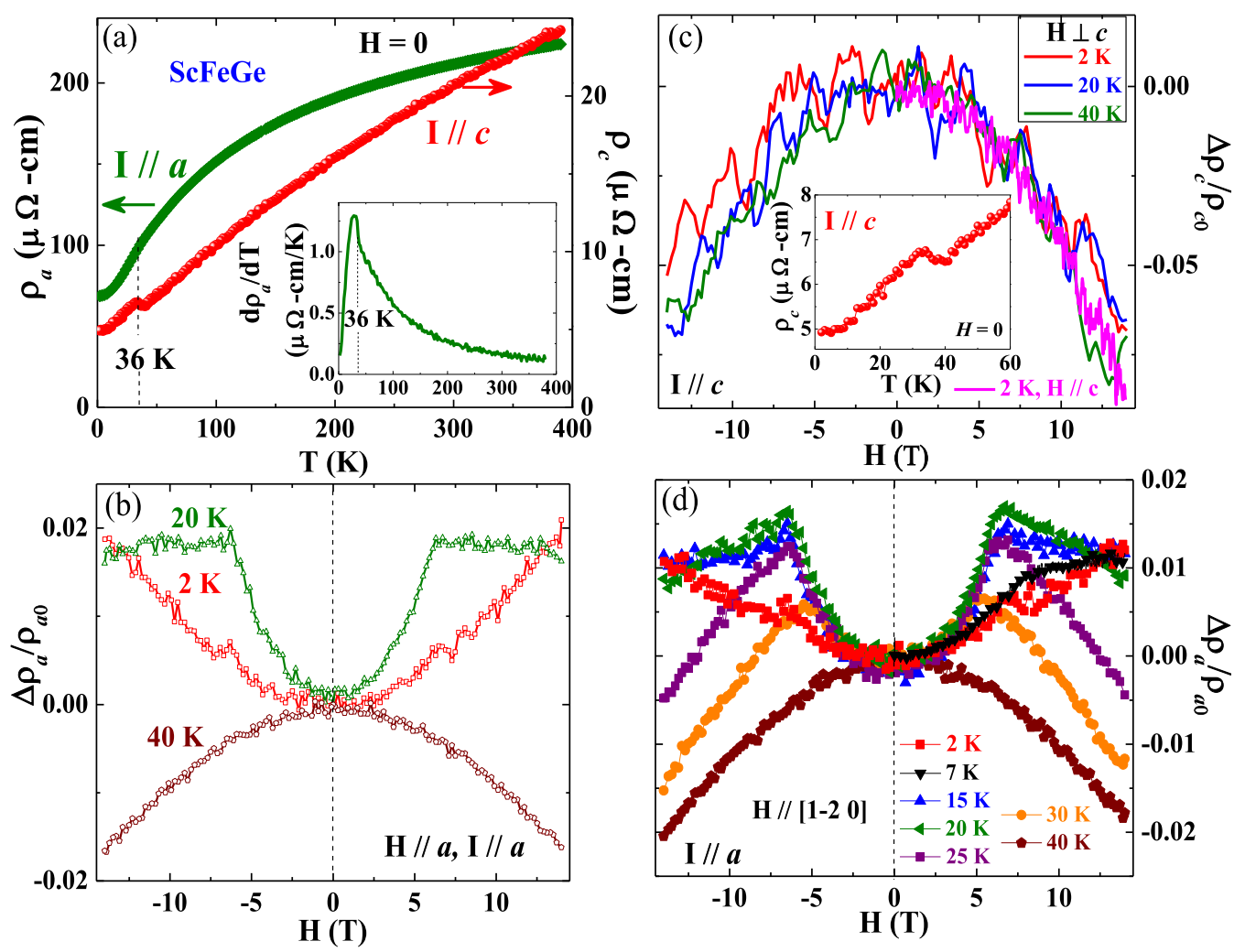

FIG. 4. Charge transport. (a) Temperature, $T$, dependence of the resistivity with the current, $I$, parallel to the $a$ axis, $\rho_{a}$, and current parallel to the $c$ axis, $\rho_{c}$, at zero field. Inset: $d \rho_{a} / d T$ vs $T$. (b) MR for current parallel to the $a$ axis, $\Delta \rho_{a} / \rho_{a}(0)=\left(\rho_{a}(H)-\rho_{a}(0)\right) / \rho_{a}(0)$, at the indicated temperatures. Data shown for $H$ parallel to the $a$ axis. (c) Magnetoresistance (MR) for current parallel to the $c$ axis, $\Delta \rho_{c} / \rho_{c}(0)=$ $\left(\rho_{c}(H)-\rho_{c}(0)\right) / \rho_{c}(0)$, at the indicated temperatures. Data are shown for field, $H$, parallel and perpendicular to the $c$ axis, as indicated in the figure. Inset: $\rho_{c}$ on an expanded scale at low $T$. (d) MR for current parallel to the $a$ axis, $\Delta \rho / \rho_{0}=\left(\rho_{a}(H)-\rho_{a}(0)\right) / \rho_{a}(0)$, at the indicated temperatures. Data shown for $H$ applied along the $\left[\begin{array}{lll}1 & \overline{2} & 0\end{array}\right]$ direction.

Fig. 3(d) so that it appears at a field of $1.9 \mathrm{~T}$ for $T=35 \mathrm{~K}$. Both $\chi(T)$ and $M(H)$ indicate an antiferromagneticlike state with a small magnetic moment apparent even in a field of $7 \mathrm{~T}$.

The specific heat at zero field is presented in Fig. 3(c), where $C_{p} / \mathrm{T}$ vs $T^{2}$ is shown. A sharp peak in $C_{p}$ at $T_{N}=36 \mathrm{~K}$ indicates that the magnetic transition discovered in $\chi(T)$ is intrinsic to $\mathrm{ScFeGe}$. This critical temperature is compatible with that found in the magnetic susceptibility as demonstrated in Fig. 3(a). We highlight the low temperature, $T<17 \mathrm{~K}, C_{p} / \mathrm{T}$ in the inset of Fig. 3(c), where a linear fit (red line) reveals an intercept, $\gamma=52.8(5) \mathrm{mJ} / \mathrm{mole}-K^{2}$ and a linear coefficient, $\beta=0.256(3) \mathrm{mJ} / \mathrm{mole}-K^{4}$. The Debye temperature $\Theta_{D}$ can be estimated using the relation $\Theta_{D}^{3}=12 n \pi^{4} k_{B} / 5 \beta$, where $n$ is the number of atoms per formula unit (f.u.), and $k_{B}$ is the Boltzmann constant yielding $\Theta_{D}=196 \mathrm{~K} . \gamma$ is significantly larger than that found in other magnetic metals with similar ordering temperatures, such as $\mathrm{MnSi}\left(T_{N}=29 \mathrm{~K}\right.$ and $\gamma=$ $32 \mathrm{~mJ} / \mathrm{mole}-K^{2}$ ), indicating larger spin-wave contributions to $C_{p}$ in $\mathrm{ScFeGe}$.

The resistivity $\rho$ of ScFeGe crystals with current oriented along the crystallographic $a\left(\rho_{a}\right)$ and $c$ axes $\left(\rho_{c}\right)$ was explored to characterize the anisotropy in the electronic properties, as well as the coupling between the magnetic and charge degrees of freedom. The temperature dependence of $\rho_{a}$ and $\rho_{c}$ at zero field are presented in Fig. 4(a), where both exhibit metallic behavior with a residual resistivity ratio (RRR) of 4.2 for $\rho_{c}$ and 3.1 for $\rho_{a} . \rho_{c}$ is found to be 13.9 times smaller than $\rho_{a}$ at low $T$, indicating a significant anisotropy in the electronic structure. There is also a significant anomaly in $\rho_{c}$ at $T_{N}$ [see inset of Fig. 4(c)], as cooling through $T_{N}$ results in a sharply increased resistivity. This suggests a significant reduction in the density of states at the Fermi level or even the opening of a partial gap in the electronic structure associated with the magnetic ordering. In contrast, $\rho_{a}$ displays only a peak in $d \rho_{a} / d T$ [Fig. 4(a)] at $T_{N}$, without any significant discontinuity apparent. We note this difference in response to the magnetic ordering in the charge conductivity along the $c$ axis and perpendicular to it indicates that the magnetic ordering affects mainly the charge carrier scattering and perhaps the electronic structure along the $c$ axis.

The magnetic field dependence of $\rho_{c}$ for $H \leqslant 14 \mathrm{~T}$ is presented in Fig. 4(c) for $H$ parallel (longitudinal magnetoresistance, MR) and perpendicular (transverse MR) to the $c$ axis. We observe a moderately sized negative MR consistent with a fluctuation scattering mechanism that is nearly independent of the field direction. In contrast, $\rho_{a}(H)$ [Figs. 4(b) and 4(d)] displays much richer behavior. Here, $\rho_{a}(H)$ is shown with $H$ along the $a$ axis (longitudinal MR) [Fig. 4(b)] and along the [1 $\overline{2}$ 0] direction (transverse MR) [Fig. 4(d)]. These are similar with only a minor variation with field direction observed. This suggests that an interaction of the charge carriers with magnetic moments, rather than an orbital effect of the field, 

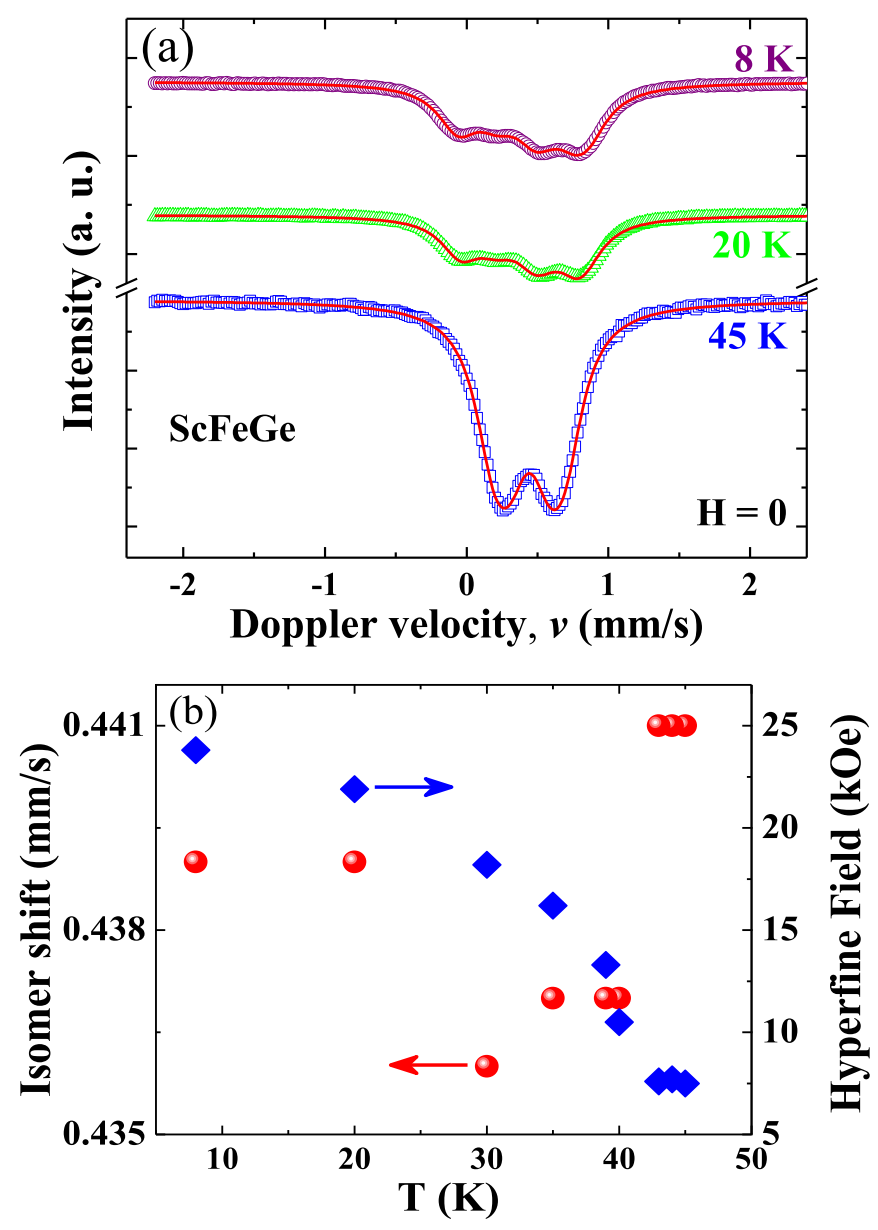

FIG. 5. Mössbauer spectroscopy. (a) Mössbauer spectra of ScFeGe collected above and below the magnetic ordering at $36 \mathrm{~K}$. (b) $T$ dependence of the isomer shift (left) and hyperfine field (right).

determines the MR. $\rho_{a}(H)$ is unusual in that there is a change in sign from a positive MR below $T_{N}$ to negative above $T_{N}$, in contrast to expectations for a simple fluctuation scattering mechanism, where a continuous suppression of scattering is common with cooling or increased $H$. The similarity of the MR for $H>H_{\mathrm{MM}}$ above and below $T_{N}$, along with the loss of the negative contribution to $\rho_{a}(H)$ at low $T$, are supportive of a fluctuation scattering mechanism as the cause of the negative MR. The positive MR, which is only observed for $T<T_{N}$, is atypical of simple metallic magnets. That it is intimately tied to the magnetic structure is made clear both by its appearance below $T_{N}$ and the change in character of the MR at the MM transition. The correspondence of the discontinuity between the positive and negative MR and the MM transition is demonstrated in Fig. 3(d). Interestingly, the discontinuity at $H_{\mathrm{MM}}$ is lost upon cooling to $2 \mathrm{~K}$. This is in contrast to the magnetization, which displays a trend toward a discontinuous transition at $H_{\mathrm{MM}}$ at low $T$. The origin of the positive MR is not yet clear.

We have also performed Mössbauer measurements to further investigate the magnetic phase transition and to probe the character of the magnetism. The Mössbauer spectra recorded from a polycrystalline ScFeGe sample is shown in Fig. 5(a). We observe a symmetric doublet at $H=0$ and $45 \mathrm{~K}$ that becomes asymmetric as the doublet splits in a hyperfine field when cooled below $T_{N}$. The shape of the spectrum at each of the three temperatures where data was collected is well represented by a fit of the model presented in the Supplemental Material [27]. The best fit parameters include a hyperfine (HF) field, which increases from 8 to $25 \mathrm{kOe}$ as $T$ is reduced from 45 to $8 \mathrm{~K}$, shown in Fig. 5(b). This small value of the hyperfine field in the magnetically ordered state is usually indicative of a small ordered magnetic moment. For example, it is much smaller than that found in $\mathrm{ScFe}_{6} \mathrm{Ge}_{6}$ formed from the same elements [46,47]. This conclusion is at odds with the effective magnetic moment of $\sim 2.3 \mu_{B}$ found from fits to $\chi$ above $T_{N}$, suggesting a difference between the ordered and fluctuating magnetic moments, as is common in weak itinerant magnets. The isomer shift is plotted in Fig. 5(b), where values similar to that found in $\mathrm{ScFe}_{6} \mathrm{Ge}_{6}[46,47]$ are displayed. The isomer shift undergoes only small variation as it is cooled through $T_{N}$, suggesting only minor changes to either the crystal or electronic structures with magnetic ordering.

$\mathrm{X}$-ray absorption near edge structure (XANES) spectroscopic measurements were performed to investigate the oxidation/electronic state of $\mathrm{Sc}, \mathrm{Fe}$, and $\mathrm{Ge}$ atoms in $\mathrm{ScFeGe}$ and to explore the possibility of differences between the sample at room temperature (RT; $T \sim 300 \mathrm{~K}$ ) and low temperature (LT; $T \sim 20 \mathrm{~K}$ ). In the case of the Fe $K$ edge [Fig. 6(a)], the near overlap of the XANES edge structure for $\mathrm{ScFeGe}$ and the metallic $\mathrm{Fe}$ standard indicates the $\mathrm{Fe}$ valence is close to zero in our compound. However, as seen in the first-derivative plot in the inset, the small inflection (indicated by the arrows), which is due to $4 s-3 d$ mixing, is energetically slightly smaller (7111 eV in ScFeGe and $7112 \mathrm{eV}$ in metallic Fe), indicating the possibility of a small reduction (corresponding to the gain of electrons) in the absorption of ScFeGe. This observation is consistent with the Bader charge analysis based on the electronic structure calculations, described below, wherein $\mathrm{Fe}$ gains 0.23 electron in $\mathrm{ScFeGe}$. For the case of $\mathrm{Sc}$, the $K$ edge in $\mathrm{ScFeGe}$ [Fig. 6(b)] is shifted with respect to the metal [Sc(0)] and the oxide [ $\mathrm{Sc}(\mathrm{III})]$. For $\mathrm{Sc}_{2} \mathrm{O}_{3}$ there is a strong pre-edge feature near $\sim 4490 \mathrm{eV}$, which can better be seen in the first-derivative plot (inset). This pre-edge structure is due to a crystal-field splitting of the Sc $3 d$ orbitals. The Sc edge for $\mathrm{ScFeGe}$ also shows a small, less intense inflection. From the maximum in the first-derivative plots, wherein the $\mathrm{Sc}(0)$ peaks at $\sim 4492 \mathrm{eV}$ and $\mathrm{Sc}(3)$ at $\sim 4503 \mathrm{eV}$, a simple linear interpolation indicates that the $\mathrm{Sc}$ in our compound (with a peak at $\sim 4496 \mathrm{eV}$ ) has an oxidation of $\sim+1.2$. This observation is also consistent with the Bader charge analysis based on the calculations described below, wherein Sc loses 1.43 electrons. Finally, in the case of Ge [Fig. 6(c)], the $\mathrm{ScFeGe} K$-edge structure appears different from the $\mathrm{Ge}(0)$ powder, despite the similarity in the edge energy position (i.e., inflection point) at $11103 \mathrm{eV}$. The oxidation of Ge is more difficult to determine from the edge energy position because of the covalent bonding. For example, although the $K$ edge is approximately $\sim 6 \mathrm{eV}$ less than that of $\mathrm{Ge}(4)$ in $\mathrm{GeO}_{2}$, other model compounds, such as GeS, have a $K$ edge at $11103 \mathrm{eV}$, much closer to our value. Because of this, it is difficult to determine the oxidation of $\mathrm{Ge}$ in our compound. The Bader charge analysis indicates that Ge gains 1.2 electrons in this compound. As seen in Figs. 6(a)-6c), the Fe, Sc, and Ge $K$ 

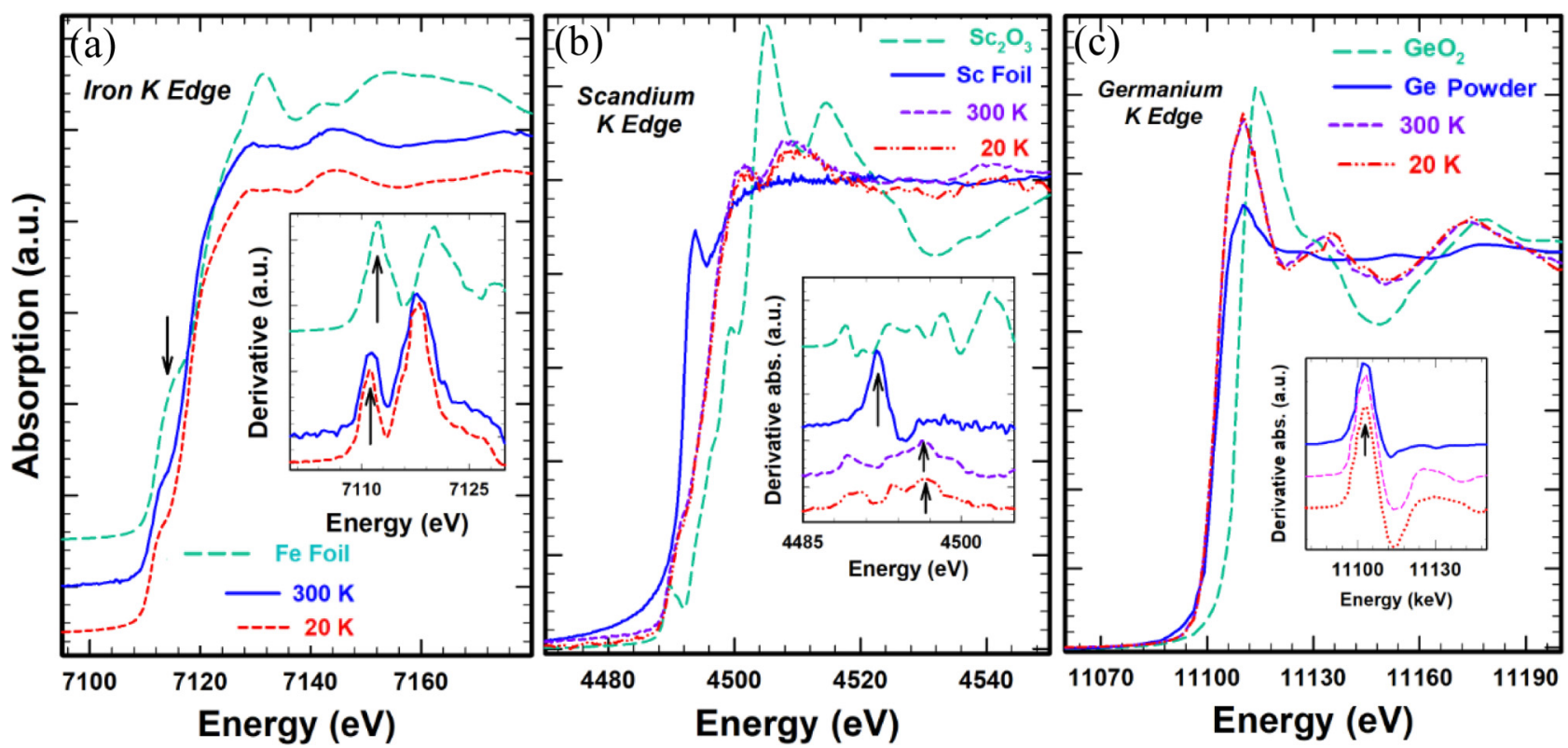

FIG. 6. X-ray absorption spectroscopy near edge structure (XANES). (a) Room temperature (RT) and low temperature (LT) (20 K) $K$-edge of Fe from $\mathrm{ScFeGe}$ (blue and red, respectively) and iron foil (Fe metal, green); inset shows the first-derivative edge positions. (b) RT and LT $K$-edge of Sc from ScFeGe (purple and red, respectively), scandium foil ( $\mathrm{Sc}$ metal, blue), and in scandia ( $\mathrm{Sc}_{2} \mathrm{O}_{3}$, green); inset shows the first-derivative edge positions. (c) RT and LT K-edge of Ge from ScFeGe (purple and red, respectively), germanium powder (Ge, blue), and germania $\left(\mathrm{GeO}_{2}\right.$, green); inset shows the first-derivative edge positions.

edges, and absorption structure of $\mathrm{ScFeGe}$, are very similar at both RT and LT, indicating no apparent oxidation changes. Thus, we are not able to resolve any significant changes to the electronic structure above and below the transition temperature $(36 \mathrm{~K})$ in our XANES measurements.

Neutron powder diffraction measurements were performed over a wide wave-vector $Q$ range to determine the magnetic structure that emerges in $\mathrm{ScFeGe}$ below $T_{N}$ (Fig. 7). Upon

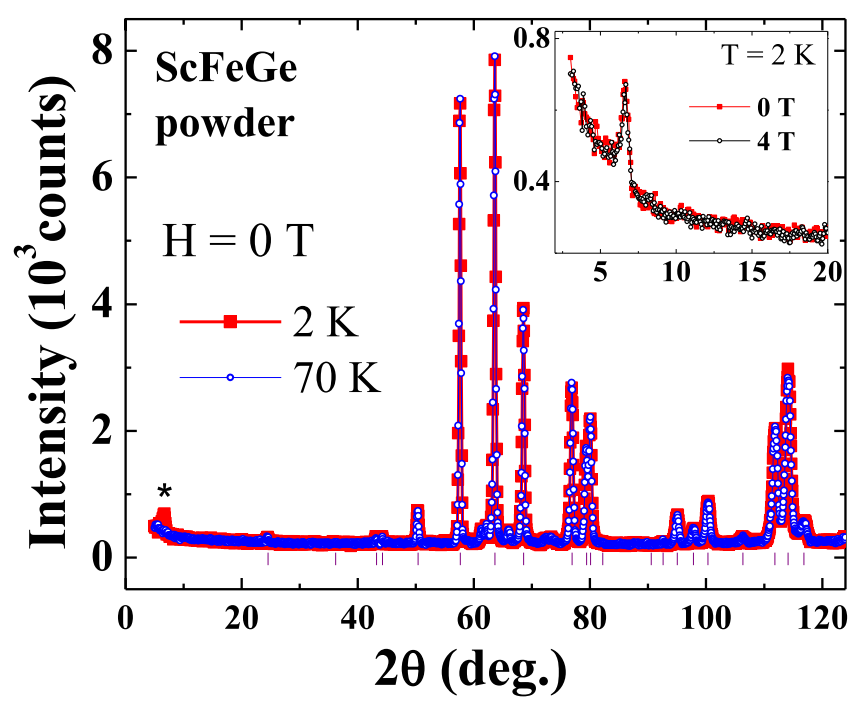

FIG. 7. Neutron powder diffraction data collected at zero field above $(70 \mathrm{~K})$ and below $(2 \mathrm{~K}) T_{N}$. Asterisk indicates the single magnetic diffraction peak. Inset shows the magnetic peak of neutron powder diffraction at $2 \mathrm{~K}$ in magnetic fields $(H)$ of 0 and $4 \mathrm{~T}$. cooling $\mathrm{ScFeGe}$ to $2 \mathrm{~K}$, a new satellite peak appears in the diffraction pattern at $2 \theta=6.5^{\circ}$ [indicated by an asterisk in Fig. 7 and in Fig. 2(b)] that was absent at 285 K. No other peaks appear above background at higher $Q$, suggesting that this additional scattering peak is magnetic in origin. In addition, we observe no change to the scattering intensity at the positions of the nuclear Bragg peaks, ruling out a substantial ferromagnetic component to the magnetic structure (see Fig. 7). The small angle at which the new scattering peak appears suggests a helical or spin density wave ordering with a pitch length or wavelength of $\sim 20 \AA$. Application of a 4-T magnetic field had no significant effect on either the angle

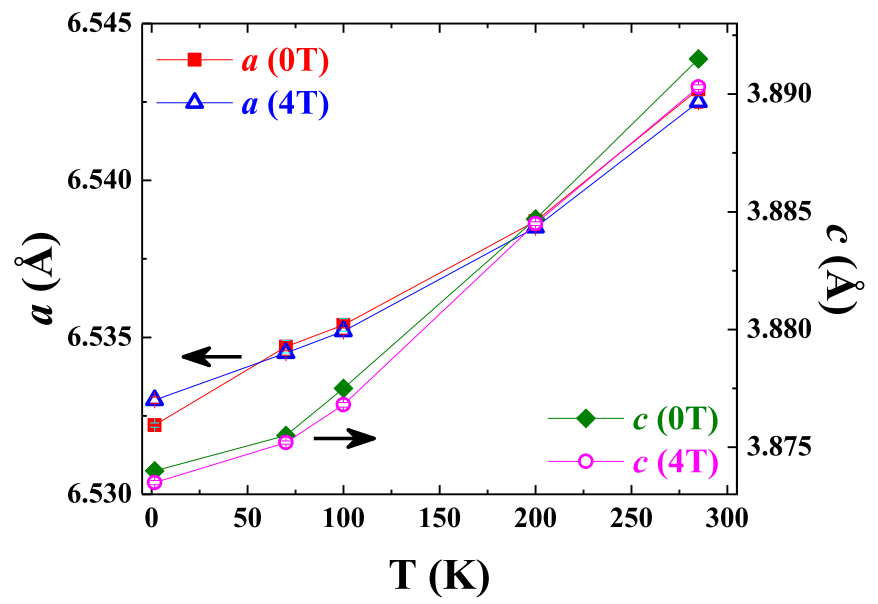

FIG. 8. Temperature $T$ dependence of the lattice parameters $a$ and $c$ at magnetic fields $H$ of 0 and $4 \mathrm{~T}$ for ScFeGe. 

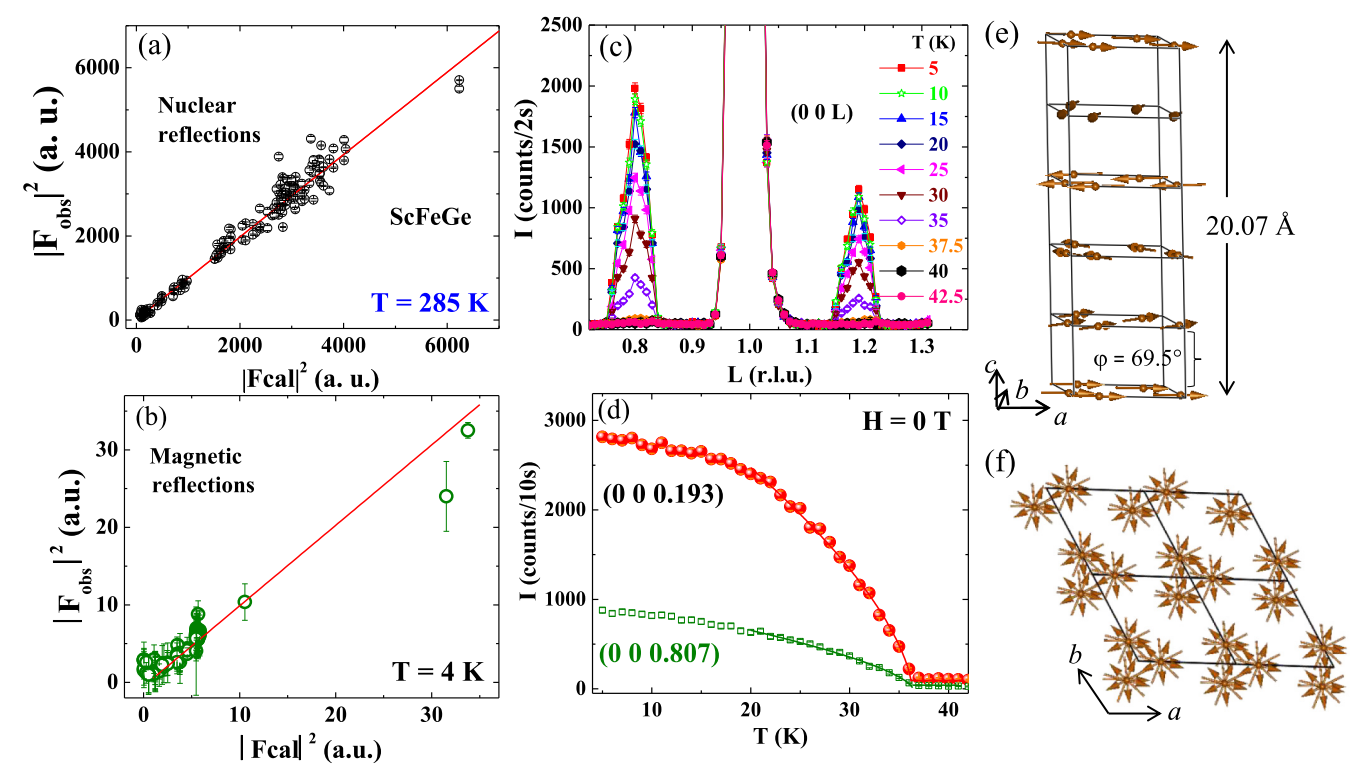

FIG. 9. Single-crystal neutron diffraction and magnetic structure. (a) Comparison of the calculated and observed values of the squared structure factors at $T=285 \mathrm{~K}$. The model for the hexagonal crystal structure with the $P \overline{6} 2 \mathrm{~m}$ space group and structural parameters determined from the PXRD at room temperature, and listed in Table S1 in the Supplemental Material [27], were used to calculate the expected intensity. (b) Comparison of the calculated and observed values of the squared structure factors for the incommensurate magnetic phase established at $T=4.0 \mathrm{~K}$ at zero field. A helical magnetic structure model was employed in the calculation. (c) $L$ scans near the $(001)$ Bragg peak at several representative temperatures collected using the four-circle diffractometer HB-3A. (d) $T$ dependence of the peak intensity of $(000.193)$ and ( $\left.\begin{array}{lll}0 & 0 & 0.807\end{array}\right)$ incommensurate magnetic peaks measured using triple-axis spectrometer HB-1A, indicating a magnetic ordering at $T_{N} \sim 36 \mathrm{~K}$. The solid line is a fit of a standard critical behavior model to the intensity (see text). (e) Schematic demonstrating the incommensurate helical magnetic structure at zero field. (f) View of the magnetic structure along the $c$ axis.

or the intensity of the magnetic scattering peak discovered (inset of Fig. 7), suggesting a much higher field is necessary to field polarize this magnetic state, in agreement with $M(H)$ measurements [Fig. 3(b)]. The temperature dependence of the lattice parameters in zero and 4-T fields, as determined from the powder neutron diffraction data, is shown in Fig. 8. No significant change in either $a$ or $c$ is observed, either as a result of the magnetic ordering at $T_{N}$ or the application of the magnetic field.

Because of the limited information provided by powder diffraction, which displayed only a single magnetic scattering peak, single-crystal neutron diffraction was carried out to establish more clearly the magnetic structure. To this end, a set of 105 reflections was collected at both 4 and $285 \mathrm{~K}$ as input for the refinement of the crystal and magnetic structures. The quality of the refinement of the crystal structure can be seen in Fig. 9(a). The refinement of the magnetic structure indicates an incommensurate helical structure along the $c$ axis with wave vector $\boldsymbol{k}=\left(\begin{array}{lll}0 & 0 & \delta\end{array}\right)$ with $\delta=0.193$. A magnetic moment of $\mu_{S}=0.53 \mu_{B}$ per Fe, confined to the $a b$ plane, was determined. The quality of the refinement is demonstrated in Fig. 9(b), and schematics of the magnetic structure are included in Figs. 9(e) and 9(f). A refinement of the magnetic scattering to a spin density wave model did not yield satisfactory results. Just as was true of the powder neutron diffraction data, we observe no discernible changes to the nuclear Bragg peaks that could be associated with a ferromagnetic component to the magnetic structure (see, e.g., Fig. S1) [27]. In addition, a number of scans along the (0
$0 \mathrm{~L}$ ) direction were performed as a function of temperature to characterize the changes to the magnetic state with warming through $T_{N}$. Figure 9 (c) presents these data in the vicinity of the $\left(\begin{array}{lll}0 & 0 & 1\end{array}\right)$ nuclear Bragg peak, where the incommensurate peaks at $\left(\begin{array}{llll}0 & 0 & 1 & -\delta\end{array}\right)$ and $\left(\begin{array}{lll}0 & 0 & 1\end{array}+\delta\right)$ are reduced with temperature, until they are indistinguishable from the background above $T_{N}$. No change in $\delta$ was observed with warming.

The temperature dependence of the $\left(\begin{array}{lll}0 & 0 & \delta\end{array}\right)$ and $\left(\begin{array}{llll}0 & 0 & 1 & -\delta\end{array}\right)$ satellite peaks is presented in Fig. 9(d) along with fits of these data to the standard form $I=I_{0}+I_{M}\left(1-T / T_{N}\right)^{2 \beta}$ as described in Ref. [48]. Here, $I_{0}$ represents the temperature independent contribution from backgrounds, $I_{M}$ is the magnetic intensity at saturation, and $\beta$ is the critical exponent. The solid lines represent the best fit of this form to the data with a value for the critical exponent $\beta=0.32$ (1) for both the $(00$ $0.193)$ and $(000.807)$ peaks at zero field. This value of $\beta$ lies between that expected for the three-dimensional Heisenberg model $(\beta=0.38)[49]$ and the two-dimensional XY model ( $\beta=0.23$ ) [50]. A correspondence of $T_{N}$ determined from this analysis with that from other measurements is displayed in Fig. 3(d).

\section{RESULTS OF FIRST-PRINCIPLES CALCULATIONS}

Electronic structure calculations were carried out to lend insight into the mechanisms for the electronic and magnetic properties presented in the previous section of this paper. The primitive cell used in our calculations was that of $\mathrm{ScFeGe}$ as described by the hexagonal lattice illustrated in Fig. 1(a). 
TABLE I. Results of electronic structure calculations for different spin orientations and exchange-correlation functionals. Lattice parameters $a$ and $c(\AA)$ and ground state energy per atom $E_{0}(\mathrm{eV} /$ atom) of ScFeGe primitive cells and supercells, obtained by including spin-orbit coupling and magnetic moment $m_{\text {spin }}\left(\mu_{B} / \mathrm{Fe}\right.$ atom). These theoretical data are compared with our experimental values measured at $2 \mathrm{~K}$.

\begin{tabular}{|c|c|c|c|c|c|}
\hline Nonmagnetic & PBE & 6.719 & 3.535 & 0.00 & -6.876 \\
\hline$c$ axis & PBE & 6.485 & 3.968 & 1.86 & -6.906 \\
\hline$a$ axis & $\begin{array}{c}\text { PBE } \\
\text { vdW-DF } \\
\text { vdW-DF2 } \\
\text { optPBE-vdW } \\
\text { optB88-vdW } \\
\text { optB86b-vdW } \\
\text { DFT-D2 } \\
\text { DFT-D3 }\end{array}$ & $\begin{array}{l}6.485 \\
6.743 \\
6.750 \\
6.629 \\
6.539 \\
6.542 \\
6.401 \\
6.409\end{array}$ & $\begin{array}{l}3.967 \\
4.057 \\
4.079 \\
4.032 \\
3.990 \\
3.991 \\
3.972 \\
3.897\end{array}$ & $\begin{array}{l}1.84 \\
2.18 \\
1.94 \\
1.97 \\
1.81 \\
1.81 \\
1.78 \\
1.52\end{array}$ & -6.906 \\
\hline $\begin{array}{l}\text { Helical-72 } \\
\text { Helical- } 90^{\circ}\end{array}$ & $\begin{array}{l}\text { PBE } \\
\text { PBE }\end{array}$ & $\begin{array}{l}6.469 \\
6.461\end{array}$ & $\begin{array}{l}3.984 \\
3.998\end{array}$ & $\begin{array}{l}1.96 \\
2.00\end{array}$ & $\begin{array}{l}-6.908 \\
-6.909\end{array}$ \\
\hline
\end{tabular}

The optimized structural parameters $(a$ and $c$ ) and the ground state energy per atom $\left(E_{0}\right)$ of $\mathrm{ScFeGe}$ that were calculated for several different spin orientations and exchange-correlation (xc) functionals are listed in Table I. For the PBE calculations, we note that the primitive cell is energetically more stable by $\sim 30 \mathrm{meV} /$ atom, when the magnetic moment is included. In our calculations we do not observe any magnetic anisotropy in the ferromagnetic state as the ground state energy is independent of the direction of the ordered moment. Therefore, to compare the differences between each xc functional, we choose to set the spin orientation along the $a$ axis. In comparison with the experimental values of bulk $\mathrm{ScFeGe}$, the PBE results along the $a$ and $c$ axis are underestimated by $0.7 \%$ $(6.485 \AA)$ and overestimated by $2.4 \%$ (3.967 $\AA$ ), respectively. When the van der Waals (vdW) corrections are included, the relative differences along the $a$ and $c$ axes increase by $3.33 \%$ $(6.750 \AA)$ and $5.29 \%(4.079 \AA)$, respectively. In the case of DFT-D3, the $a$ axis is compressed by around $1.9 \%$ (6.409 $\AA$ ), while the inclusion of the long-range $\mathrm{vdW}$ interactions expand the $c$ axis by only $0.6 \%(3.897 \AA)$. Taking the measured lattice constants from $\mathrm{ScFeGe}$ as a reference suggests that the semilocal PBE functional is the best option to calculate the physical properties of $\mathrm{ScFeGe}$. The calculated magnetic moments $m_{\text {spin }}$ for ScFeGe are also summarized in Table I. We notice that all calculated magnetic moments deviate substantially from the experimental values for the fluctuating effective magnetic moment at $T>T_{N}\left(\sim 2.3 \mu_{\mathrm{B}} /\right.$ f.u. $)$ and the ordered magnetic moment determined from refinements of the neutron diffraction data $\left(\mu_{S}=0.53 \mu_{B} / \mathrm{Fe}\right)$.

The electronic band structure of nonmagnetic ScFeGe was calculated employing the PBE functional, as shown in Fig. 10(a) for energies up to $1 \mathrm{eV}$ above and below Fermi energy. There are several nearly degenerate flat bands (highlighted in red) located just above the Fermi level along the high-symmetry $\boldsymbol{k}$ path of A-L-H-A. This contour delimits the upper edge of the first Brillouin zone (BZ), as shown in Fig. 10(b) [51]. In addition, several valence and conduction bands cross the Fermi level mainly along $\Gamma-\mathrm{A}$, L-M, and K-H lines, i.e., along the $k_{z}$ axis. The orbital-character analysis was performed to establish the character of the bands closest to the Fermi level. As shown in Fig. 11, the $d$ orbitals of Fe,

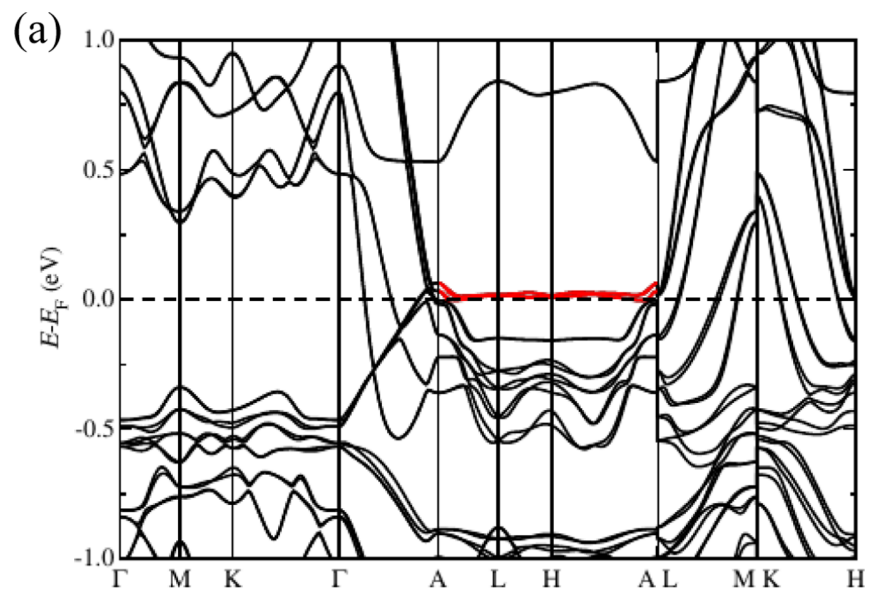

(b)

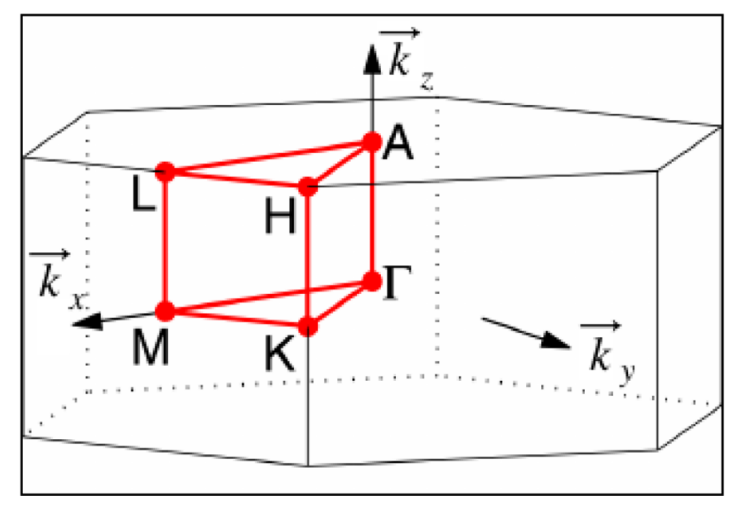

FIG. 10. Band structure. (a) Calculated electronic band structure of nonmagnetic ScFeGe for energies $E$ within $1 \mathrm{eV}$ of the Fermi energy $E_{F}$. Spin-orbit coupling is included. $E_{F}$ is at zero energy. Highlighted in red are the nearly degenerate flat bands near $E_{F}$. (b) The first Brillouin zone of a hexagonal lattice [51]. 
(a)

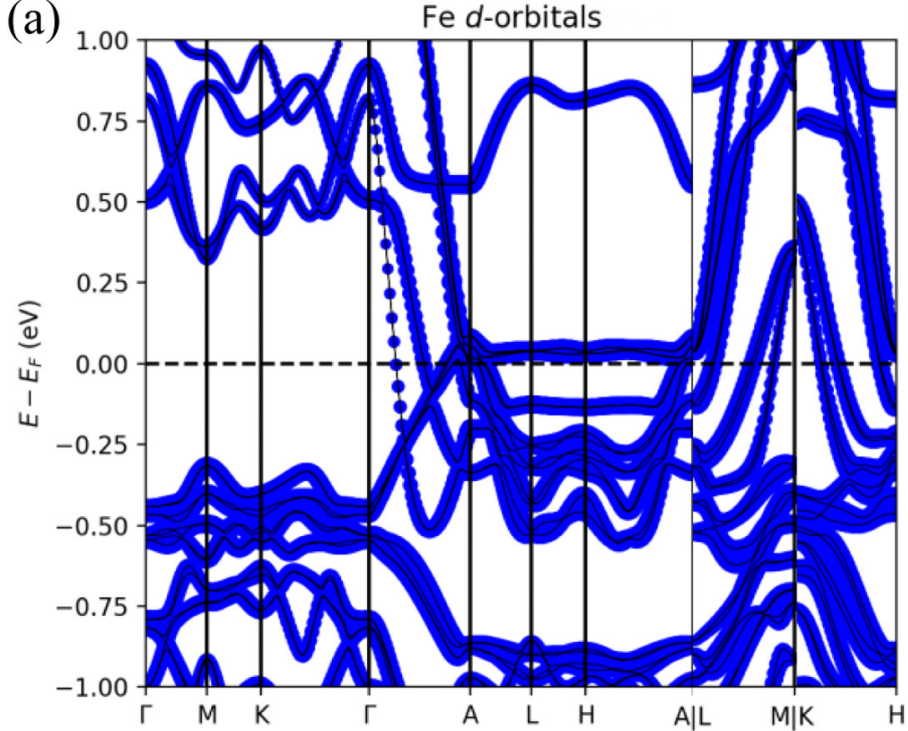

(b)

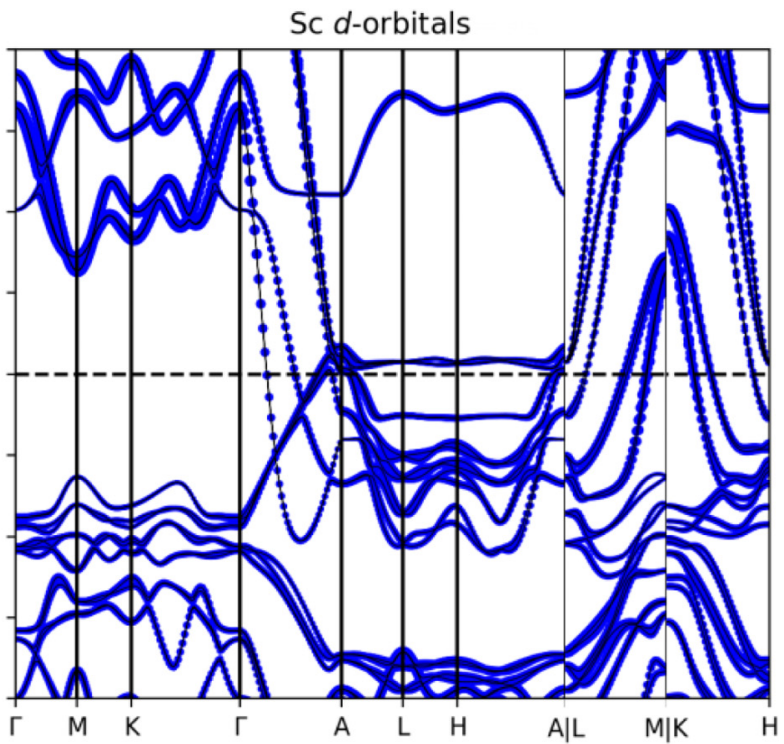

(c)

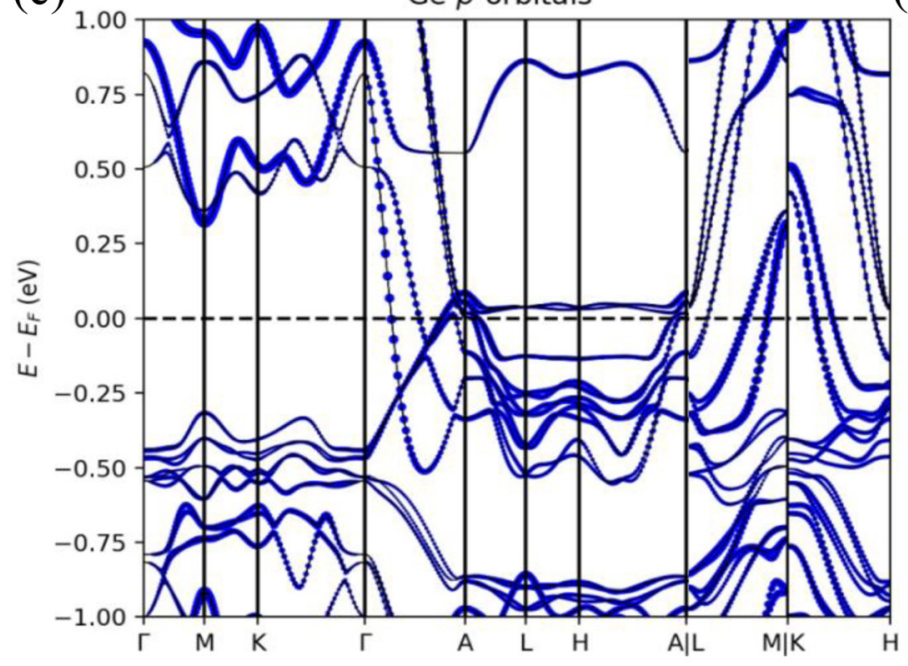

(d)

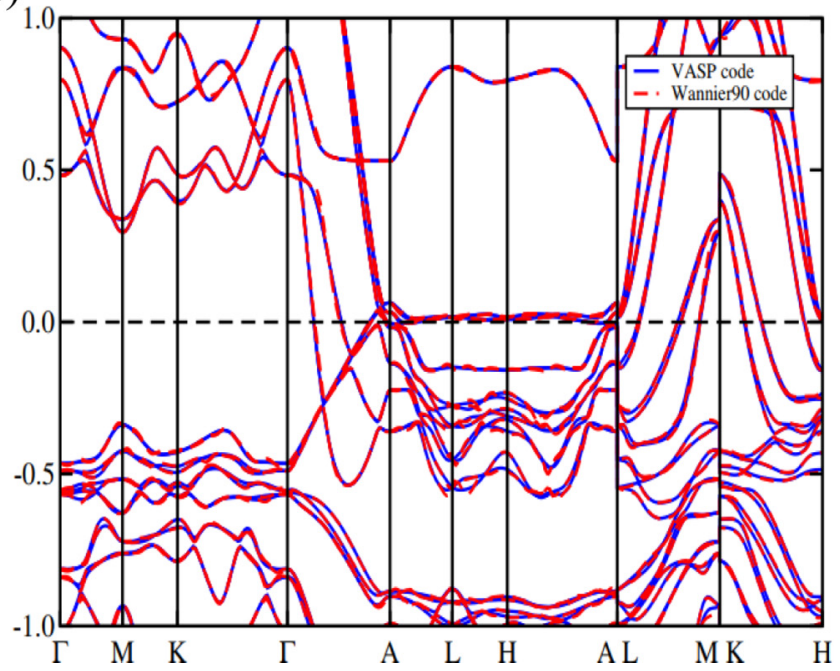

FIG. 11. Calculated electronic band structure of nonmagnetic ScFeGe showing orbitals of (a) Fe, (b) Sc, and (c) Ge in which $d$-type orbitals of the Fe atoms are the dominant feature for these bands. The size of the filled circles are proportional to the weight projected onto the orbitals. (d) Calculated electronic band structure of nonmagnetic ScFeGe, using first-principles calculations (blue line) and Wannier functions (red dashed line), where Kohn-Sham states are projected onto Fe- $d$, Sc- $d$, and Ge- $p$ localized orbitals. The spin-orbit coupling is included. The Fermi level is at zero energy.

and in particular the $d_{z^{2}}$ state, are dominant in the vicinity of the Fermi level. In addition, the electronic bands located between 0.5 and $1 \mathrm{eV}$ above the Fermi level derive mainly from Sc- $d$ and Ge- $p$ orbitals. A Bader charge analysis was performed that showed that Fe gains 0.23 electron, Ge gains 1.20 electrons, and Sc loses 1.43 electrons. These are in general agreement with the XANES results, but we note that the XANES result for Ge was inconclusive.

The nearly degenerate flat bands close to the Fermi level suggest an electron-driven instability. One way to explore this possibility is to investigate the three-dimensional (3D) Fermi surface sheets (FSs). These were determined from the symmetrical Wannier tight-binding model to project the Kohn-Sham states onto Fe- $d$, Sc- $d$, and Ge- $p$ localized orbitals. The resulting electronic band structure is compared to that obtained from first-principles calculations in Fig. 11(d), where it can be seen that the interpolated bands reproduce the electronic structure of $\mathrm{ScFeGe}$ in the range between -1 and $1 \mathrm{eV}$ very well. The resulting Fermi surfaces are depicted in Figs. 12(a) and 12(b), showing nearly flat sheets perpendicular to the $k_{z}$ axis, as suggested by the band structure of Fig. 10(a), along with two ellipsoidal Fermi surface sheets centered at the top and bottom of the BZ. For better visibility, we project the FS onto the $\left[\begin{array}{llll}1 & 0 & 1 & 0\end{array}\right]$ plane, as shown in Fig. 12(c). Along the $\Gamma$-A line, we can extract two wave vectors, $\boldsymbol{k}_{z_{1}}=\left(\begin{array}{lll}0 & 0 & 0.262\end{array}\right)$ and $\boldsymbol{k}_{z_{2}}=\left(\begin{array}{lll}0 & 0 & 0.191\end{array}\right)$, which connect the three flattest Fermi surface sheets. The magnitude of these vectors, particularly $\boldsymbol{k}_{z_{2}}$, is similar to the helical wave vector obtained from neutron diffraction. $\boldsymbol{k}_{z_{1}}$ and $\boldsymbol{k}_{z_{2}}$ can be associated with turn angles of $94.32^{\circ}$ and $68.76^{\circ}$ between moments in adjacent planes. Figure 12(d) shows three cuts through the FSs perpendicular to $\boldsymbol{k}_{z}$ at $\boldsymbol{k}_{z}=-0.136,-0.265$, and -0.456 . It is clear 
(a)

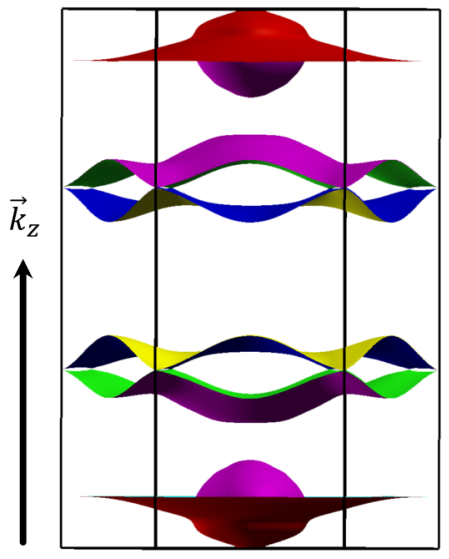

(b)

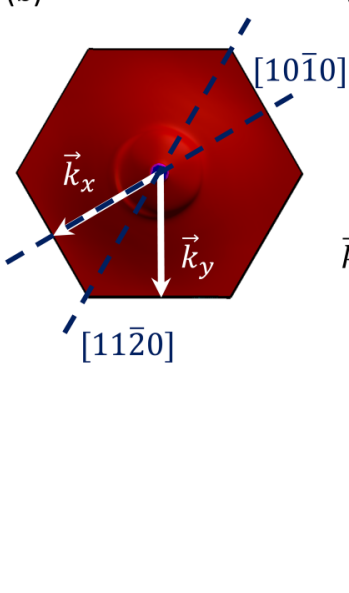

(c)

(d)

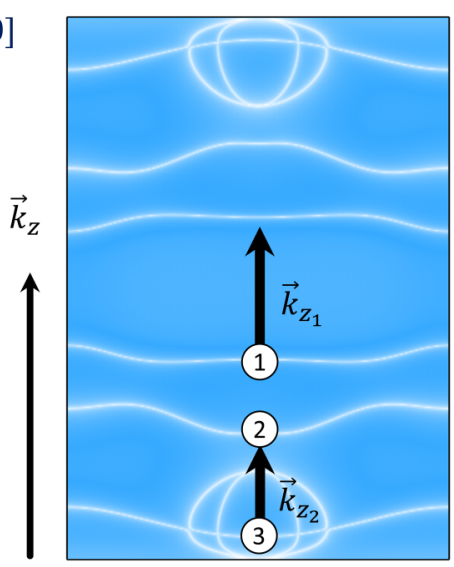

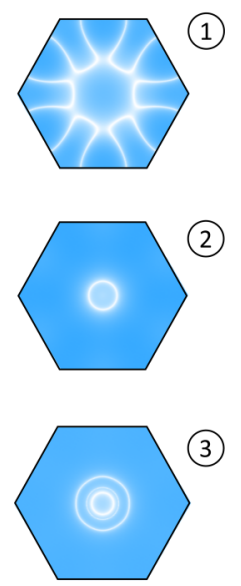

FIG. 12. Fermi surface. (a) Side and (b) top views of the calculated three-dimensional Fermi surface (FS) sheets in the first Brillouin zone for nonmagnetic bulk ScFeGe. (c) Calculated bulk FSs on a cleaved $\left[\begin{array}{llll}1 & 0 & \overline{1} & 0\end{array}\right]$ surface. The coordinates of $k_{z_{1}}$ and $k_{z_{2}}$ are $\left(\begin{array}{llllll}0 & 0 & 0.262\end{array}\right)$ and $\left(\begin{array}{ll}0 & 0\end{array}\right.$ 0.191), respectively. (d) Bulk FSs at $k_{z}=-0.136,-0.265$, and -0.456 and labeled as (1), (2), and (3), respectively. The spin-orbit coupling is included.

that first-principles calculations predict a highly nested Fermi surface with nesting wave vectors similar to the incommensurate wave vector found in neutron diffraction, although our calculations overestimate the size of the moments in the magnetically ordered state. This, along with the anomaly observed in the resistivity for currents along the $c$ axis, strongly suggests a Fermi surface nesting instability as the mechanism driving the magnetic phase transition discovered in $\mathrm{ScFeGe}$.

To determine the stability of helical magnetic order at both $\boldsymbol{k}_{z_{1}}$ and $\boldsymbol{k}_{z_{2}}$, we have considered $(1 \times 1 \times 4)\left(90^{\circ}\right.$ moment rotation between adjacent planes $)$ and $(1 \times 1 \times 5)\left(72^{\circ}\right.$ rotation) supercells, as shown in Figs. 13(a) and 13(b), respectively. These are commensurate structures that we use

(a)

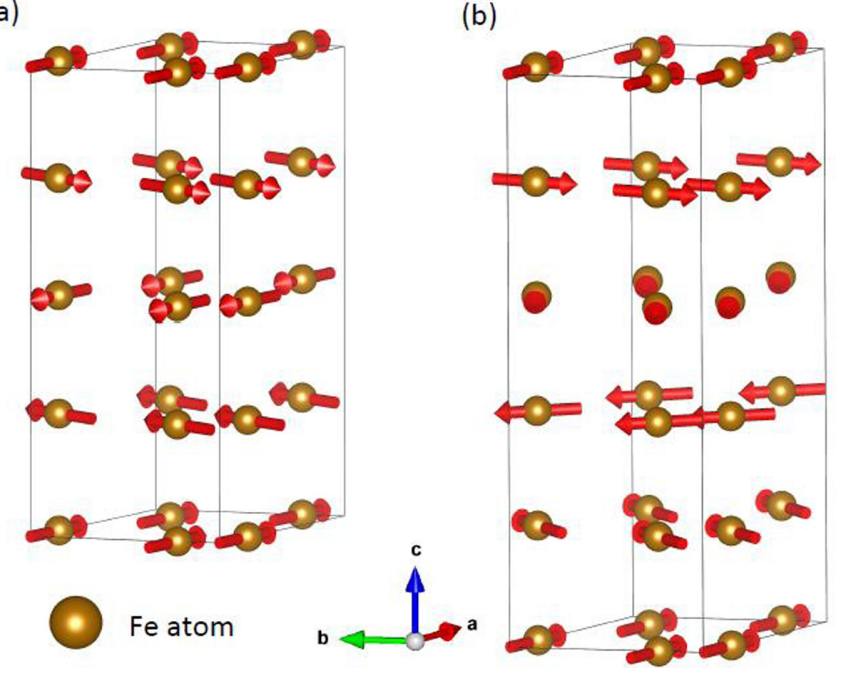

FIG. 13. Schematics of the supercells used in the (a) helical $90^{\circ}(1 \times 1 \times 4)$ supercell and $(\mathrm{b})$ helical-72 $(1 \times 1 \times 5)$ supercell calculations. For clarity, only the iron atoms are shown with the spin orientations represented by red arrows. to approximate the predicted incommensurate ordering associated with the Fermi surface nesting to allow calculations to be performed. The optimized structural parameters for these calculations are included in Table I. Both supercell structures behave in a similar fashion in that the $a$ axis decreases $(\sim 0.25 \%)$, while the $c$ axis increases $(\sim 0.78 \%)$, when compared with the calculated primitive cell of $\mathrm{ScFeGe}$. This indicates that the noncollinearity of the spin orientation strongly influences the ScFeGe structure. Energetically, these two structures are equivalent to within our computational accuracy at $-6.909 \mathrm{eV} /$ atom, i.e., at zero temperature the $90^{\circ}$ - and $72^{\circ}$-helical structures are nearly degenerate. Note that for each of these helical structures, we find that a spin orientation where the spins reside in the plane perpendicular to $\boldsymbol{q}$ is energetically preferred. If instead, we restrict the moment to the $a c$ plane we find a structure that is less stable by $3 \mathrm{meV}$. Consequently, we find that the helical state $(\boldsymbol{k} \| c$ with moments in the $a b$ plane) is energetically favorable compared to the cycloid state $(\boldsymbol{k} \| c$ with magnetic moments in the $a c$ plane) in agreement with the magnetic structure determined via neutron diffraction. For a finite transition temperature, the free energy, including the entropy, must be considered to determine which of these structures may be more stable. If we consider a Ginzburg-Landau expansion in powers of the Fourier components of the magnetization $m_{k}$, the implication of the $a b$ initio calculations are that the coefficients of the $\left|m_{\boldsymbol{k}}\right|^{2}$ terms for both $\boldsymbol{k}_{z}=\left(\begin{array}{lll}0 & 0 & 0.25\end{array}\right)$ and $\boldsymbol{k}_{z}=\left(\begin{array}{lll}0 & 0 & 0.2\end{array}\right)$ change sign at approximately the same temperature. Since these leading instabilities compete for the same magnetic moment, their interaction is repulsive. If we assume that the energy landscape in proximity to the nesting vectors is not too steep, then the result of this calculation is that there are closely competing helical magnetic states in ScFeGe. Given that the neutron scattering data supports a single- $\boldsymbol{k}$ structure rather than multi- $\boldsymbol{k}$ structure, the repulsive interaction between the two orders is likely sufficiently strong to prevent coexistence of both types [52,53]. In that case, the actual transition temperature will be renormalized by the thermal fluctuations of the 
respective order parameters. In mode-mode coupling theory $[54,55]$, the thermal fluctuations are determined by the values of the coefficients of the quartic terms in the expansion and by the phase space available for fluctuations in the vicinity of $\boldsymbol{k}_{z_{1}}$ and $\boldsymbol{k}_{z_{2}}$. The information on these coefficients is not contained in the $a b$ initio results, and one may plausibly argue that the lower symmetry $72^{\circ}$ results in a sufficiently large phase space for fluctuations to bring the transition temperature below that of the $90^{\circ}$ order.

\section{DISCUSSION AND CONCLUSIONS}

In summary, we have discovered magnetic ordering in NCS hexagonal ScFeGe at $T_{N}=36 \mathrm{~K}$ apparent in the magnetic susceptibility, magnetization, specific heat, electrical transport, Mössbauer spectroscopy, and neutron diffraction measurements, as summarized in the phase diagram of Fig. 3(d). In addition, a metamagnetic transition is found (at $6.7 \mathrm{~T}$ for $T=$ $2 \mathrm{~K}$ ), when the field is oriented within the hexagonal $a b$ plane but absent for $H$ parallel to the $c$ axis. Our neutron diffraction measurements reveal an incommensurate helimagnetically ordered state below $T_{N}$ with a wave vector $\boldsymbol{k}=\left(\begin{array}{lll}0 & 0 & 0.193\end{array}\right)$ and a magnetic moment of $\mu_{S}=0.53 \mu_{B} / \mathrm{Fe}$ aligned within the $a b$ plane. The size of $\mu_{S}$ is in reasonable agreement with the small hyperfine field observed in our Mössbauer measurement but inconsistent with the effective magnetic moment determined from the Curie constant ( $\mu_{\text {eff }} \sim 2.3 \mu_{B} /$ f.u.). The ratio of these moments, $\mathrm{RW}=\mu_{\text {eff }} / \mu_{S}$, is known as the RhodesWohlfarth parameter and is used to indicate the degree of itinerancy of the magnetic state [56]. Here, RW $=4.3$ is comparable to that observed in famous itinerant magnets $\mathrm{ZrZn}_{2}$ [48,57,58], MnSi [48,59], and $\mathrm{Fe}_{1-x} \mathrm{Co}_{x} \mathrm{Si}[60]$, indicating that $\mathrm{ScFeGe}$ belongs to the class of weak itinerant magnets. This is important, as such systems are typically sensitive to chemical substitution and pressure. As a result, many of these systems display quantum criticality with the possibility of unconventional superconductivity or other exotic phases. The tendency of our polycrystalline samples with an increased Ge content to display higher $T_{N}$ is consistent with this expectation.

The strong connection of the magnetism with the charge carriers is reflected in the MR as well. The MR displays unusual field and temperature dependencies for $T<T_{N}$ including the appearance of a positive MR below $T_{N}$ and at fields $H<H_{\mathrm{MM}}$ for $H$ oriented perpendicular to the $c$ axis. The implication is that these fields cause an increase in scattering for the charge carriers and suggests a complex variation of the magnetic structure with field. In addition to the experimental data, our electronic structure calculations indicate an itinerant mechanism for the magnetic ordering in the form of a strongly nested Fermi surface with one nesting vector consistent with the neutron diffraction data.

The helimagnetic ordering with a wave vector along the $c$ axis in a hexagonal system draws natural comparisons to the heavy rare earth elemental metals Tb, Dy, and Ho. These materials display incommensurate helimagnetic states over a finite temperature range below the critical temperature where there is a strong temperature dependence to the helimagnetic pitch length [20]. The ground states are ferromagnetic, as $H_{\mathrm{MM}}$ is reduced to zero field with cooling or there is a tran- sition to a commensurate conical phase $[61,62]$. In addition, nesting conditions along the $c$ axis have been discovered in electronic structure calculations suggesting a strong connection of the magnetism to the charge degrees of freedom [63]. These materials have drawn more recent attention because of the complex magnetic structures that form when exposed to magnetic fields oriented perpendicular to the $c$ axis [20]. Here, fan, helifan, and spin-slip states have been discovered with abrupt transitions in magnetic fields [20,64]. There are also significant differences between these heavy rare earth metals and $\mathrm{ScFeGe}$, including the character of the magnetism, as $f$-electron materials are thought of as having localized magnetic moments that interact via the conducting charge carriers. ScFeGe has a small ordered magnetic moment, so that a local magnetic moment model is not appropriate, and we have demonstrated that the helimagnetic pitch is not strongly temperature or field dependent. Despite these differences, the experience with the heavy rare earth elemental metals suggests complex magnetic structures may exist at finite field in $\mathrm{ScFeGe}$. Thus, how the magnetic state of $\mathrm{ScFeGe}$ approaches the field polarized state at higher magnetic fields is an interesting unanswered question. The path to full polarization may be substantially different in ScFeGe than the classic cases discussed for the rare earth elemental metals based upon the weak itinerant character of its magnetism.

Although the motivation for this work was to explore a NCS magnet where the DMI is expected to play a role, it appears that the DMI may play only a minor role in the mechanism for helimagnetism in ScFeGe. In addition, helimagnetism is often described to occur due to competing interactions where there are exchange interactions of opposing sign and similar magnitudes. In this case, the expectation is that magnetic fields cause a distortion of the helical state causing regions where the magnetic moments are mostly along the field to grow at the expense of regions where the moments oppose the applied field $[65,66]$. For materials that are well described by this model, a MM transition is found to occur to a magnetic fan state with $H_{\mathrm{MM}} \sim 0.5 H_{\text {sat }}$, where $H_{\text {sat }}$ is the field necessary to saturate the magnetization $[67,68]$. Instead, for $\mathrm{ScFeGe}$, we find that the pitch of the helical state is determined by the electronic structure rather than these two common mechanisms, competing exchange interactions, or a significant DMI.

Thus, the role of the DMI in ScFeGe has not been resolved in our measurements or our calculations, thus far. This is important, since materials with hexagonal symmetry and significant DMI interaction have yielded helical magnets with magnetic solitonic states and unusual magnetic domain structures that evolve with field $[6,8]$. Coupled to this unanswered question is the question of topological protection in a polar noncentrosymmetric helimagnet. In this case, the magnetic ordering breaks mirror symmetry, creating the possibility of topologically relevant solitonic states that persist in both fields and thermal fluctuations, and with domains of both left- and right-handed helical magnetic ordering allowed by symmetry. It is unknown if a single domain state can be nucleated or controlled resulting in a truly topologically relevant state. The data presented here suggest that $\mathrm{ScFeGe}$ is a weakly itinerant magnet with interesting coupling between the charge and magnetic degrees of freedom that make it an ideal material to 
investigate domain walls and changes to the domain structures with application of a magnetic field.

\section{ACKNOWLEDGMENTS}

The experimental material presented here is supported by the US Department of Energy under EPSCoR Grant No. DESC0012432 with additional support from the Louisiana Board of Regents. This research used resources at the High Flux Isotope Reactor, a DOE Office of Science User Facility operated by the Oak Ridge National Laboratory. Part of this work was performed at the Swiss Spallation Neutron Source SINQ, Paul Scherrer Institut, Villigen, Switzerland. The computational work conducted by W.A.S. and D.T. was also supported by the US Department of Energy under EPSCoR Grant No. DESC0012432 with additional support from the Louisiana Board of Regents. I.V. acknowledges support from NSF Grant No. DMR 1410741 for theoretical work. Part of this work was performed using supercomputing resources provided by the Center for Computation and Technology (CCT) at Louisiana State University and the Center for Computational Innovations (CCI) at Rensselaer Polytechnic Institute.
[1] U. K. Rößler, A. N. Bogdanov, and C. Pfleiderer, Nature (London) 442, 797 (2006).

[2] S. Mühlbauer, B. Binz, F. Jonietz, C. Pfleiderer, A. Rosch, A. Neubauer, R. Georgii, and P. Boni, Science 323, 915 (2009).

[3] X. Z. Yu, Y. Onose, N. Kanazawa, J. H. Park, J. H. Han, Y. Matsui, N. Nagaosa, and Y. Tokura, Nature (London) 465, 901 (2010).

[4] X. Z. Yu, N. Kanazawa, Y. Onose, K. Kimoto, W. Z. Zhang, S. Ishiwata, Y. Matsui, and Y. Tokura, Nat. Mater 10, 106 (2011).

[5] N. Nagaosa and Y. Tokura, Nat. Nanotechnol. 8, 899 (2013).

[6] Y. Togawa, T. Koyama, K. Takayanagi, S. Mori, Y. Kousaka, J. Akimitsu, S. Nishihara, K. Inoue, A. S. Ovchinnikov, and J. Kishine, Phys. Rev. Lett. 108, 107202 (2012).

[7] N. J. Ghimire, M. A. McGuire, D. S. Parker, B. Sipos, S. Tang, J.-Q. Yan, B. C. Sales, and D. Mandrus, Phys. Rev. B 87, 104403 (2013).

[8] S. K. Karna, F. N. Womack, R. Chapai, D. P. Young, M. Marshall, W. Xie, D. Graf, Y. Wu, H. Cao, L. DeBeer-Schmitt, P. W. Adams, R. Jin, and J. F. DiTusa, Phys. Rev. B 100, 184413 (2019).

[9] E. B. Myers, D. C. Ralph, J. A. Katine, R. N. Louie, and R. A. Buhrman, Science 285, 867 (1999).

[10] C. Tannous, and R. L. Comstock, Springer Handbook of Electronic and Photonic Materials (Springer, Cham, 2017).

[11] S. Seki, X. Z. Yu, S. Ishiwata, and Y. Tokura, Science 336, 198 (2012)

[12] Y. Tokunaga, X. Z. Yu, J. S. White, H. M. Ronnow, D. Morikawa, Y. Taguchi, and Y. Tokura, Nat. Commun. 6, 7638 (2015).

[13] I. Kézsmárki, S. Bordács, P. Milde, E. Neuber, L. M. Eng, J. S. White, H. M. Rønnow, C. D. Dewhurst, M. Mochizuki, K. Yanai, H. Nakamura, D. Ehlers, V. Tsurkan, and A. Loidl, Nat. Mater 14, 1116 (2015).

[14] T. Kurumaji, T. Nakajima, V. Ukleev, A. Feoktystov, T.-h. Arima, K. Kakurai, and Y. Tokura, Phys. Rev. Lett. 119, 237201 (2017).

[15] S. V. Grigoriev, D. Chernyshov, V. A. Dyadkin, V. Dmitriev, S. V. Maleyev, E. V. Moskvin, D. Menzel, J. Schoenes, and H. Eckerlebe, Phys. Rev. Lett. 102, 037204 (2009).

[16] T. Tanigaki, K. Shibata, N. Kanazawa, X. Yu, Y. Onose, H. S. Park, D. Shindo, and Y. Tokura, Nano Lett. 15, 5438 (2015).

[17] I. Koretsune, N. Nagosa, and R. Arita, Sci. Rep. 5, 13302 (2015).

[18] A. Fert, V. Cros, and J. Sampaio, Nat. Nanotechnol. 8, 152 (2013).
[19] G. Finocchio, F. Büttner, R. Tomasello, M. Carpentieri, and M. Kläui, J. Phys. D: Appl. Phys. 49, 423001 (2016).

[20] T. Chattopadhyay, Science 264, 226 (1994).

[21] B. Y. Kotur and R. I. Andrusyak, Dopovidi Akademii Nauk Ukranskoi RSR Seriya B-Geologichni Khimichni Ta Biologichni Nauki 12, 40 (1984).

[22] D. M. Liu, Q. Z. Huang, M. Yue, J. W. Lynn, L. J. Liu, Y. Chen, Z. H. Wu, and J. X. Zhang, Phys. Rev. B 80, 174415 (2009).

[23] O. Tegus, E. Brück, K. H. J. Buschow, and F. R. de Boer, Nature 415, 150 (2002).

[24] R. V. Skolozdra, B. Ya. Kotur, R. I. Andrusyak, and Yu. K. Gorelenko, Izvestiya Akademii Nauk SSSR, Neorganicheskie Materialy 27:8, 1632 (1991).

[25] A. C. Larson and R. B. Von Dreele, LANL Report LA-UR-86748 (1990).

[26] H. M. Rietveld, J. Appl. Crystallogr. 2, 65 (1969).

[27] See Supplemental Material at http://link.aps.org/supplemental/ 10.1103/PhysRevB.103.014443 for details on the structural refinement.

[28] B. C. Chakoumakos, H. B. Cao, F. Ye, A. D. Stoica, M. Popovici, M. Sundaram, W. Zhou, J. S. Hicks, G. W. Lynn, and R. A. Riedel, J. Appl. Cryst. 44, 655 (2011).

[29] J. Rodrguez-Carvajal, Physica B (Amsterdam) 192, 55 (1993).

[30] D. J. Haynes, D. Shekhawat, D. Berry, A. Roy, and J. J. Spivey, J. Rare Earths 38, 711 (2019).

[31] L. Macheli, A. Roy, E. Carleschi, B. P. Doyle, E. van, Catal. Today 343, 176 (2020).

[32] B. Ravel and M. Newville, J. Synchrotron Radiat. 12, 537 (2005).

[33] G. Kresse and J. Hafner, Phys. Rev. B 47, 558 (1993).

[34] G. Kresse and J. Hafner, Phys. Rev. B 49, 14251 (1994).

[35] G. Kresse and J. Furthmuller, Phys. Rev. B 54, 11169 (1996).

[36] G. Kresse and J. Furthmuller, Comput. Mater. Sci. 6, 15 (1996).

[37] P. E. Blochl, Phys. Rev. B 50, 17953 (1994).

[38] G. Kresse and D. Joubert, Phys. Rev. B 59, 1758 (1999).

[39] J. Klimes, D. R. Bowler, and A. Michaelides, J. Phys. Condens. Matter 22, 022201 (2009).

[40] J. Klimes, D. R. Bowler, and A. Michaelides, Phys. Rev. B 83, 195131 (2011).

[41] T. Bucko, J. Hafner, S. Lebegue, and J. G. Angyan, J. Phys. Chem. A 114, 11814 (2010).

[42] S. Grimme, J. Antony, S. Ehrlich, and H. A. Krieg, J. Chem. Phys. 132, 154104 (2010).

[43] A. A. Mostofi, J. R. Yates, G. Pizzi, Y.-S. Lee, I. Souza, D. Vanderbilt, and N. Marzari, Comput. Phys. Commun. 185, 2309 (2014). 
[44] G. Pizzi, V. Vitale, R. Arita, S. Blügel, F. Freimuth, G. Geranton, M. Gibertini, D. Gresch, C. Johnson, T. Koretsune et al., J. Phys.: Condens. Matter 32, 165902 (2020).

[45] Q. Wu, S. Zhang, H.-F. Song, M. Troyer, and A. A. Soluyanov, Comput. Phys. Commun. 224, 405 (2018).

[46] T. Mazet, O. Isnardb, and B. Malamana, Solid State Commun. 114, 91 (2000).

[47] S. F. Matar, T. Fickenscher, B. Gerke, O. Niehaus, U. Ch. Rodewald, A. F. Al Alam, N. Ouaini, and R. Pottgen, Solid State Sci. (Elsevier) 39, 82 (2015).

[48] T. Chatterji, Neutron Scattering from Magnetic Materials (Elsevier, Amsterdam, 2006).

[49] K. Ghosh, C. J. Lobb, R. L. Greene, S. G. Karabashev, D. A. Shulyatev, A. A. Arsenov, and Y. Mukovskii, Phys. Rev. Lett. 81, 4740 (1998).

[50] J. Als-Nielsen, S. T. Bramwell, M. T. Hutchings, G. J. McIntyre, and D. Visser, J. Phys.: Condens. Matter 5, 7871 (1993).

[51] W. Setyawan and S. Curtarolo, Comput. Mater. Sci. 49, 299 (2010).

[52] K.-S. Liu and M. E. Fisher, J. Low. Temp. Phys. 10, 655 (1973).

[53] J. M. Kosterlitz, D. R. Nelson, and M. E. Fisher, Phys. Rev. B 13, 412 (1976).

[54] K. K. Murata and S. Doniach, Phys. Rev. Lett. 29, 285 (1972).
[55] G. G. Lonzarich and L. Taillefer, J. Phys. C: Solid State Phys. 18, 4339 (1985).

[56] P. Rhodes and E. Wohlfarth, Proc. R. Soc. A 273, 247 (1963).

[57] S. J. Pickart, H. A. Alperin, G. Shirane, and R. Nathans, Phys. Rev. Lett. 12, 444 (1964).

[58] E. A. Yelland, S. J. C. Yates, O. Taylor, A. Griffiths, S. M. Hayden, and A. Carrington, Phys. Rev. B 72, 184436 (2005).

[59] C. Pffeiderer, S. R. Julian, and G. G. Lonzarich, Nature (London) 414, 427 (2001).

[60] S. Kawarazaki, H. Yasuoka, Y. Nakamura, and J. H. Wernick, J. Phys. Soc. Jpn. 41, 1171 (1976).

[61] K. M. Döbrich, A. Bostwick, J. L. McChesney, K. Rossnagel, E. Rotenberg, and G. Kaindl, Phys. Rev. Lett. 104, 246401 (2010).

[62] E. Mendive-Tapia and J. B. Staunton, Phys. Rev. Lett. 118, 197202 (2017).

[63] W. E. Evenson and S. H. Liu, Phys. Rev. 178, 783 (1969).

[64] J. Jensen and A.R. Mackintosh, Phys. Rev. Lett. 64, 2699 (1990).

[65] L. Udvardi, S. Khmelevskyi, L. Szunyogh, P. Mohn, and P. Weinberger, Phys. Rev. B 73, 104446 (2006).

[66] J. K. Glasbrenner, K. M. Bussmann, and I. I. Mazin, Phys. Rev. B 90, 144421 (2014).

[67] P. A. Herpin and P. Meriel, J. Phys. Radium 22, 337 (1961).

[68] T. Nagamiya, K. Nagata, and Y. Kitano, Prog. Theor. Phys. 27, 1253 (1962). 